OPEN ACCESS

Edited by:

Mingli Liu,

Morehouse School of Medicine,

United States

Reviewed by:

Yumin Wang,

Central South University, China

Juni Sarkar,

University of Southern California,

Los Angeles, United States

*Correspondence:
Mohammad Haris
mharis@sidra.org
IkhlakAhmed
iahmed2@sidra.org

${ }^{\dagger}$ These authors have contributed equally to this work and share first authorship

¥These authors share senior authorship

Specialty section:

This article was submitted to

Molecular Medicine,

a section of the journal

Frontiers in Cell and Developmental

Biology

Received: 14 October 2020

Accepted: 04 January 2021

Published: 05 February 2021

Citation:

Nisar S, Bhat AA, Singh $M$ Karedath T, Rizwan A, Hashem S, Bagga P, Reddy R, Jamal F, Uddin S, Chand G, Bedognetti D, El-Rifai W,

Frenneaux MP, Macha MA, Ahmed and Haris M (2021) Insights Into the Role of CircRNAs: Biogenesis,

Characterization, Functional, and Clinical Impact in Human

Malignancies.

Front. Cell Dev. Biol. 9:617281. doi: 10.3389/fcell.2021.617281

\section{Insights Into the Role of CircRNAs: Biogenesis, Characterization, Functional, and Clinical Impact in Human Malignancies}

\author{
Sabah Nisar ${ }^{1+}$, Ajaz A. Bhat ${ }^{1 \dagger}$, Mayank Singh ${ }^{2 t}$, Thasni Karedath ${ }^{3 \dagger}$, Arshi Rizwan ${ }^{4}$, \\ Sheema Hashem ${ }^{7}$, Puneet Bagga ${ }^{5}$, Ravinder Reddy ${ }^{6}$, Farrukh Jamal ${ }^{7}$, Shahab Uddin ${ }^{8}$, \\ Gyan Chand ${ }^{9}$, Davide Bedognetti ${ }^{10,11,12}$, Wael El-Rifai ${ }^{13}$, Michael P. Frenneaux ${ }^{14}$, \\ Muzafar A. Macha ${ }^{15}$, Ikhlak Ahmed ${ }^{3,16 * \pm}$ and Mohammad Haris ${ }^{1,17 * \neq}$
}

${ }^{1}$ Functional and Molecular Imaging Laboratory, Cancer Research Department, Sidra Medicine, Doha, Qatar, ${ }^{2}$ Dr. B. R. Ambedkar Institute Rotary Cancer Hospital (BRAIRCH), All India Institute of Medical Sciences (AlIMS), New Delhi, India, ${ }^{3}$ Research Branch, Sidra Medicine, Doha, Qatar, ${ }^{4}$ Department of Nephrology, All India Institute of Medical Sciences (AlIMS), New Delhi, India, ${ }^{5}$ Diagnostic Imaging, St. Jude Children's Research Hospital, Memphis, TN, United States, ${ }^{6}$ Department of Radiology, Perelman School of Medicine at the University of Pennsylvania, Philadelphia, PA, United States,

${ }^{7}$ Dr. Rammanohar Lohia Avadh University, Ayodhya, India, ${ }^{8}$ Translational Research Institute, Academic Health System, Hamad Medical Corporation, Doha, Qatar, ${ }^{9}$ Department of Endocrine Surgery, Sanjay Gandhi Post Graduate Institute of Medical Sciences, Lucknow, India, ${ }^{10}$ Laboratory of Cancer Immunogenomics, Cancer Research Department, Sidra Medicine, Doha, Qatar, " ${ }^{12}$ College of Health and Life Sciences, Hamad Bin Khalifa University, Doha, Qatar, ${ }^{13}$ Department of Surgery, University of Miami Miller School of Medicine, Miami, FL, United States, ${ }^{14}$ Academic Health System, Hamad Medical Corporation, Doha, Qatar, ${ }^{15}$ Watson-Crick Centre for Molecular Medicine, Islamic University of Science and Technology (IUST), Pulwama, India, ${ }^{16}$ Center for Interdisciplinary Research and Innovations, University of Kashmir, Srinagar, India, ${ }^{17}$ Laboratory Animal Research Center, Qatar University, Doha, Qatar

Circular RNAs (circRNAs) are an evolutionarily conserved novel class of non-coding endogenous RNAs (ncRNAs) found in the eukaryotic transcriptome, originally believed to be aberrant RNA splicing by-products with decreased functionality. However, recent advances in high-throughput genomic technology have allowed circRNAs to be characterized in detail and revealed their role in controlling various biological and molecular processes, the most essential being gene regulation. Because of the structural stability, high expression, availability of microRNA (miRNA) binding sites and tissue-specific expression, circRNAs have become hot topic of research in RNA biology. Compared to the linear RNA, circRNAs are produced differentially by backsplicing exons or lariat introns from a pre-messenger RNA (mRNA) forming a covalently closed loop structure missing $3^{\prime}$ poly-(A) tail or $5^{\prime}$ cap, rendering them immune to exonucleasemediated degradation. Emerging research has identified multifaceted roles of circRNAs as miRNA and RNA binding protein (RBP) sponges and transcription, translation, and splicing event regulators. CircRNAs have been involved in many human illnesses, including cancer and neurodegenerative disorders such as Alzheimer's and Parkinson's disease, due to their aberrant expression in different pathological conditions. The 
functional versatility exhibited by circRNAs enables them to serve as potential diagnostic or predictive biomarkers for various diseases. This review discusses the properties, characterization, profiling, and the diverse molecular mechanisms of circRNAs and their use as potential therapeutic targets in different human malignancies.

Keywords: circRNA, RNA binding protein, miRNA sponges, signaling pathways, tumor, drug resistance

\section{INTRODUCTION}

Circular RNAs (circRNAs) are single-stranded non-coding RNAs that are covalently linked to form a continuous closed-loop and participate in the regulation of transcriptional and posttranscriptional gene expression (Wang M. et al., 2017). In recent years, circRNA has become a hotspot in research due to its ability to regulate a myriad of processes that include transcription, translation, splicing and sequestering RNA binding proteins (RBPs) and microRNAs (miRNAs) from their targets (Bartsch et al., 2018). Apart from the widely accepted role of circRNAs as miRNA sponges, circRNAs are also found to act as protein sponges, scaffolds, decoys and recruiters (Huang A. et al., 2020). Studies have found that circRNAs promote tumor progression in cancers such as lung adenocarcinoma, gastric cancer and cervical cancer by acting as RNA sponge and binding to miRNA, thereby increasing downstream gene expression (Tang Q. et al., 2019; Zhang X. et al., 2019; Xu Y. et al., 2020). CircRNAs are formed in the circular transcript by backsplicing of premature messenger RNAs (mRNAs). During the transcription process in eukaryotic cells, there is always a competition between linear and backsplicing. The presence of long introns, RBPs and inverted repeat elements favor the backsplicing event during transcription, and the splice-donor site downstream is brought closer to the splice-acceptor site upstream either by RBP dimerization or by base pairing between inverted repeat elements (Kristensen et al., 2019). The backsplicing event can result in the formation of different types of circRNA such as exon-intron circRNA (ElcircRNAs) (consists of both introns and exons), circular intronic RNAs (formed by introns), exonic circRNA (formed by the splicing of introns), and tRNA intronic circRNA (formed by pre-tRNA splicing) (Zhao X. et al., 2019). CircRNAs are presumably more stable than linear RNA because of the lack of $5^{\prime}$ and $3^{\prime}$ ends, and ribonucleases do not easily digest them. The short half-life of linear RNA can be overcome by constructing engineered circRNAs and cyclizing mRNA, thereby promoting stable protein expression in eukaryotic cells (Wesselhoeft et al., 2018). The expression of circRNAs is disrupted in a wide range of diseases, including cancer. They have been proposed as potential biomarkers for cancer therapy as circRNAs can be easily detected in the patients' blood plasma (Wu Q. et al., 2019). circRNAs regulate cancer progression and are involved in various cancer signaling pathways such as PI3K/AKT, MAPL/ERK1/2, and $\mathrm{Wnt} / \beta$-catenin signaling pathways due to their interaction with miRNAs (Yang Z. et al., 2017). The aberrant translation of circRNAs alters tumor malignancy, and in addition to the many described functions of circRNA, they can also be retrotranscribed and function as competitive RNA (Dong et al., 2016).
Circular RNAs were initially thought to be unable to translate through cap-dependent mechanisms due to their lack of $5^{\prime}$ cap structure and poly-A tail. But recent studies have shown the ability of circRNAs to translate in prokaryotes by mimicking DNA rolling circle amplification and association of circRNAs with translating ribosomes and the ability of circRNAs to generate proteins from circRNA minigenes (Abe et al., 2013; Pamudurti et al., 2017). The translations of circRNAs can be classified as an internal ribosome entry site (IRES) independent dependent and IRES dependent (Tatomer and Wilusz, 2017). IRES-independent translations are found in the circRNAs present in the HeLa cells (Abe et al., 2015). While IRES-dependent translations require additional non-canonical cellular factors to recruit ribosomes to the IRES element and are found in circZNF609 as the UTR element of circZNF609 drives the IRES-dependent translation process through splicing event (Legnini et al., 2017). Capdependent translation is inefficient and inhibited under stress conditions or viral infections. In contrast, mRNA translation can be initiated by an IRES-mediated cap-independent mechanism, which is known to be unaltered by these unfavorable conditions (Yang and Wang, 2019).

\section{BIOGENESIS OF CircRNAs}

Circular RNAs are produced by non- canonical splicing events commonly known as backsplicing, which is considered an alternative splicing event. Although back splicing is regarded as an alternative splicing event, the molecular mechanism underlying the circular RNAs' biogenesis remains elusive (Mao et al., 2018). CircRNAs are derived from canonical splice sites and depend on canonical splicing machinery, which is usually inefficient to generate linear RNAs (Salzman et al., 2012; Jeck et al., 2013; Memczak et al., 2013; Ashwal-Fluss et al., 2014). On the contrary, results obtained from studies on Drosophila showed inhibition of spliceosome components by U2snRNP depletion or inhibition that caused increased circRNA generation compared to its linear counterparts (Liang D. et al., 2017). Hence it is proven that, when pre-mRNA processing events are halted, nascent RNA can be redirected to different alternative pathways that can facilitate back splicing and, ultimately, circRNA generation (Kramer et al., 2015; Liang D. et al., 2017). Apart from the defective splicing machinery, looping of flanking intron sequences on both sides of exons, namely splice donor site and splice acceptor site can support efficient circularization of diverse exons across eukaryotes (Kramer et al., 2015). The looping can be mediated by base pairing of Alu repeats or any inverted repeat elements located in the upstream and downstream introns 
(Ivanov et al., 2015; Kelly et al., 2015; Figure 1). Other mechanisms that facilitate backsplicing are the dimerization of RBPs like Quaking or FUS; those are known to bind in the specific motifs of the flanking introns (Conn et al., 2015; Errichelli et al., 2017; Verheijen and Pasterkamp, 2017). Even though most circRNAs are exonic, a large group of circRNA having long introns flanking the exons are involved in backsplicing (Jeck et al., 2013). These intron-exon circRNAs are usually derived from genes with highly active promoters (Enuka et al., 2016; Ferreira et al., 2018; Kristensen et al., 2018). Evidence suggests that most circular RNAs' biogenesis was influenced by cis-acting elements and trans-acting splice factors (Kramer et al., 2015). Moreover, during the exon skipping process, lariat formation events can lead to the formation of intronic circRNA under specific circumstances like escaping from the debranching process of lariats (Kelly et al., 2015; Robic et al., 2020). Additionally, epigenetic modifications within the histones and gene bodies affect alternative splicing and directly impact circRNA biogenesis (Shukla et al., 2011; Bentley, 2014). These findings suggest that even though the biogenesis of circRNA is a product of inefficient canonical splicing, the introns within the circRNAs mostly spliced out during the process indicates biogenesis is instead a straightforward regulatory process orchestrated in the splicing machinery (Westholm et al., 2014).

\section{PROPERTIES AND CHARACTERIZATION OF CircRNAs}

After the biogenesis of circRNAs, most of the exonic circRNAs transport to the cytoplasm, while the intronic and intron-exon circRNAs remain in the nucleus. The transportation of circRNAs is carried out in a size-dependent manner by ATP dependent RNA helicase URH49 (for shorter circles) and spliceosome RNA helicase UAP56 (for longer circles) (Huang et al., 2018). Exonic circRNAs generally have a low level of expression compared to their parental linear counterparts, except for a few whose expression levels are independent of their parental mRNA (Hsu and Coca-Prados, 1979; Capel et al., 1993; You et al., 2015; Holdt et al., 2016; Cortés-López et al., 2018; Vo et al., 2019). CircRNAs are exceptionally stable due to lack of free ends like mRNAs and are resistant to exonuclease digestion (Jeck et al., 2013; Memczak et al., 2013; Enuka et al., 2016). The turnover of circles is mostly mediated by N6-methyladonosine (m6A), and circles are subjected to the RNAse-P-multidrug associated protein (MRP) complex induced endonuclease cleavage (Liu C.X. et al., 2019). Another turnover mechanism is associated with miRNA regulated argonaute 2 (AGO2) protein-mediated cleavage, as shown in AGO2-miR7miR-671 silencing complex for degradation of ciRS-7 (Hammond et al., 2001; Kleaveland et al., 2018). CircRNAs are enriched in exosomes or extracellular vesicles, suggesting that these extracellular vesicles might be a possible clearance mechanism or might serve as facilitators of the cell to cell communication through circles (Dou et al., 2016; Lasda and Parker, 2016; Preußer et al., 2018).

\section{DISCOVERY AND PROFILING OF CircRNAs}

Circular RNAs are mostly evolutionary conserved and often expressed in cell-type, tissue-specific or developmental stages, specifically in organisms (Memczak et al., 2013). RNA sequencing in ribosomal RNA (rRNA) depleted total RNA leads to the discovery of many circRNAs in cancer cells, heart tissues and brain cells through a specific bioinformatics pipeline to identify back splicing junctions (Salzman et al., 2013; Maass et al., 2017). Individual circRNA identification and validations are explained in Table 1. Apart from the genome-wide proofing of circRNA using RNA sequencing or microarray analysis locus-specific circRNA profiling, validations can be done in a locus-specific manner using different methods, as explained in Table 2. The circRNAs can be visualized by RNA in situ hybridization and is a useful technique for understanding its biology by co-localizing potential miRNA sponges (You et al., 2015; Han et al., 2017).

\section{IDENTIFICATION OF CircRNAs FROM RNA-SEQ DATA USING BIOINFORMATICS APPROACH}

Several computational methods have been developed over the last few years to predict circRNA structures and their expression levels from rRNA-depleted or total RNA sequenced libraries (Table 1). RNA library preparation protocols can profile circRNA reads to varying extents. While methods such as polyA+ selection can result in a depleted representation of circRNAs (Jakobi and Dieterich, 2019), treatment with RNAse-R can enrich circRNA reads in the RNA-Seq data (Ahmed et al., 2016, 2019). The computational detection of circRNA expression requires a high sequencing yield from the high-throughput Next Generation Sequencing run as most circRNAs are expressed at low levels. Longer reads and paired-end libraries perform better for reliable detection of circRNA structure and estimation of expression. Pre-processing of RNA-Seq data such as removal of adaptor sequences or additional in silico flittering for rRNA reads can lead to performance enhancements of circRNA detection algorithms (Hansen, 2018; Jakobi and Dieterich, 2018). Indeed, all computational algorithms for circRNA detection use the unique morphology of circRNAs in which exons are atypically joined in a non-linear order through a head-to-tail "backsplice," to identify chimeric reads that support the existence of the backsplice junction (BSJ). However, chimeric reads can also originate from multiple other sources such as genomic rearrangements of exonic sequences, tandem duplications, template switching from reverse transcription, chimeric amplification or trans-splicing (Ahmed et al., 2016; Jakobi and Dieterich, 2019). Although chimeric reads are the only reads that directly confirm the existence of a BSJ, the orientation of mapped paired-end reads across exonjunctions could be used in addition to inferring the presence of the BSJ. Thus, for each circRNA isoform, the expression can be summarized as the aggregate total number of chimeric and supportive reads that align to the exons in divergent orientation 


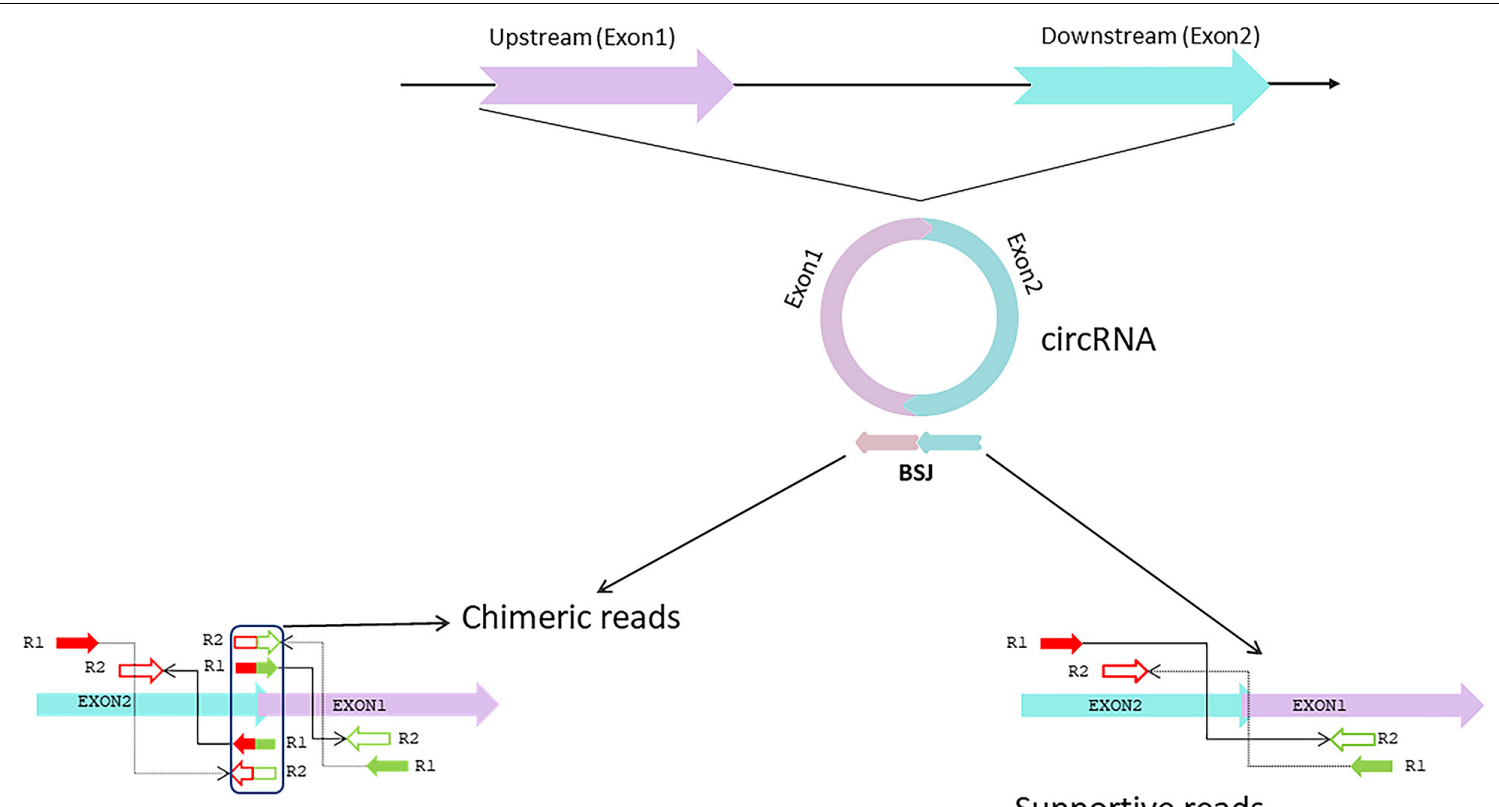

Supportive reads

FIGURE 1 | Biogenesis of circular RNA. The figure represents three different circular RNAs; Intron -exon, exon and intron circular RNAs, and its biogenesis mechanism during pre-mRNA splicing events. Intron pairing takes place with repeated inverted elements like Alu repeats, whereas lariat-driven biogenesis produces circRNAs during the exon skipping process. circRNAs can produce functional proteins that directly impact tumor progression (a few examples are shown in the box). circRNAs also act as protein sponges and are involved in protein recruitment.

TABLE 1 | Selected published tools for circRNA detection and expression analysis.

\begin{tabular}{|c|c|c|}
\hline Tool name & Software link & References \\
\hline find_circ & https://github.com/marvin-jens/find_circ & Memczak et al., 2013 \\
\hline CIRCexplorer & https://github.com/YangLab/CIRCexplorer & Zhang et al., 2014 \\
\hline circRNA_finder & https://github.com/orzechoj/circRNA_finder & Westholm et al., 2014 \\
\hline ACFS & https://github.com/arthuryxt/acfs & You et al., 2015 \\
\hline NCLscan & https://github.com/TreesLab/NCLscan & Chuang et al., 2016 \\
\hline PTESFinder & https://sourceforge.net/projects/ptesfinder-v1/ & Izuogu et al., 2016 \\
\hline UROBORUS & https://github.com/WGLab/UROBORUS & Song et al., 2016 \\
\hline miARma & https://sourceforge.net/projects/miarma/ & Andrés-León et al., 2016 \\
\hline circseq-cup & https://github.com/bioinplant/circseq-cup/ & Ye et al., 2017 \\
\hline AutoCirc & https://github.com/chanzhou/AutoCirc & Zhou et al., 2017 \\
\hline circTools & http://starbase.sysu.edu.cn/circTools.php & Zhang and Yang, 2018 \\
\hline STARChip & https://github.com/LosicLab/STARChip & Akers et al., 2018 \\
\hline CirComPara & http://github.com/egaffo/CirComPara & Gaffo et al., 2017 \\
\hline CircRNAFisher & https://github.com/duolinwang/CircRNAFisher & Jia et al., 2019 \\
\hline CircMarker & https://github.com/lxwgcool/CircMarker & Li et al., $2018 \mathrm{~g}$ \\
\hline circtools & https://github.com/dieterich-lab/circtools & Jakobi et al., 2019 \\
\hline NCLcomparator & https://github.com/TreesLab/NCLcomparator & Chen and Chuang, 2019 \\
\hline $\mathrm{CIRI}$ & https://sourceforge.net/projects/ciri/ & Zheng Y. et al., 2019 \\
\hline CircRNAwrap & https://github.com/liaoscience/circRNAwrap & Li L. et al., 2019 \\
\hline Ularcirc & https://github.com/VCCRI/Ularcirc & Humphreys et al., 2019 \\
\hline Docker4Circ & https://github.com/kendomaniac/docker4seq & Ferrero et al., 2019 \\
\hline
\end{tabular}




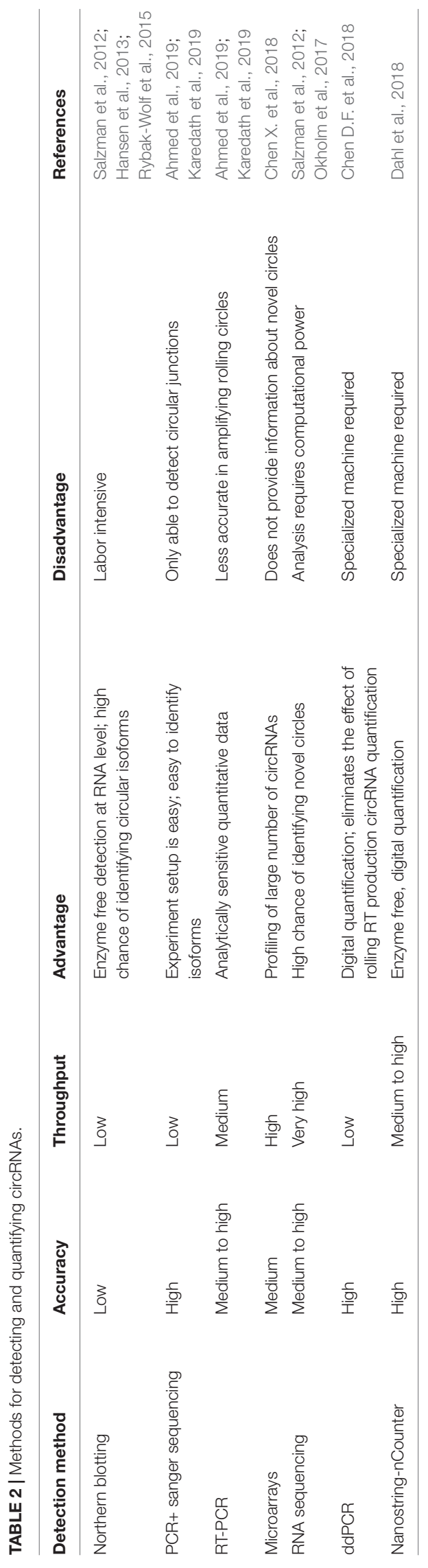

with respect to the direction of transcription, suggesting the presence of a BSJ instead of a linear junction (Figure 2).

Software tools for circRNA detection from RNA-Seq data use various approaches to identify BSJ and assess its expression (Table 1). While some methods use custom-scripts to analyze the data generated by the standard read mapping tools such as Bowtie2 (Langmead and Salzberg, 2012), TopHat2 (Kim et al., 2013), or STAR (Dobin et al., 2013), others have improved read alignment tools for circRNA detection with minor post-processing required (Hoffmann et al., 2014). Docker images have recently been developed that provide an endto-end solution in a modular framework for multiple aspects of circRNA expression analysis such as circRNA prediction, classification, annotation, sequence analysis, and differential expression analysis (Gaffo et al., 2017; Ferrero et al., 2019). Also, multiple data resources have been built that provide annotation and functional information on circRNAs (Glažar et al., 2014; Chen et al., 2016; Xia et al., 2017; Li et al., 2018f; Meng et al., 2018) and their association with diseases and traits (Ghosal et al., 2013; Xia S. et al., 2018; Yao et al., 2018).

\section{FUNCTIONAL ROLE OF CircRNAs WITH SPECIAL EMPHASIS ON CircRNA-PROTEIN INTERACTION IN TUMOR CELLS}

Circular RNAs have an important non-coding function as they retained the third position of codons, which is redundant and highly conserved in many circles (Memczak et al., 2013). The biological function of circRNAs is not yet fully identified except that a few circles act as miRNA sponges to regulate downstream target genes (Hansen et al., 2013; Memczak et al., 2013; Zheng et al., 2016; Piwecka et al., 2017). However, most circRNAs do not have multiple sponging sites that can effectively sponge miRNAs but contain protein binding sites (Ashwal-Fluss et al., 2014). For example, circRNAs viz. circMbl, circFOXO3, and circANRIL are involved in protein regulatory functions (Huang A. et al., 2020). Meanwhile, circPABPN1, located in the cytoplasm, acts as a protein decoy or a sponge to HuR, thereby suppressing PABPN1 gene translation and reducing cellular proliferation (Abdelmohsen et al., 2017). Some circRNAs act as protein scaffolds to regulate its parental gene function (Huang A. et al., 2020). CircFOXO3 acts as a protein scaffold that facilitates MDM2 independent ubiquitylation of p53 and acts as a sponge for MDM2, thereby preventing ubiquitination of its parental gene FOXO3 (Du et al., 2017a). These processes are highly implicated in cancer as the presence of circFOXO3 can induce stress-induced apoptosis and reduce cell viability (Du et al., 2017b). A study found that circAmotl1, a circRNA that is highly expressed in neonatal cardiac tissues, was involved in the cardioprotective role by binding to phosphoinositide-dependent kinase-1 (PDK1) and AKT-1 and facilitating the nuclear translocation of pAKT (Du et al., 2017a). Friend leukemia virus integration 1 (FLI1), an ETS transcription factor family member that acts as an oncogenic driver in hematological malignancies was found to 
enhance the invasiveness of breast cancer cells (MDA-MDB 231) by binding to the FLI1 promoter and recruiting TET1 (demethylase), thereby regulating DNA demethylation (Chen N. et al., 2018). Studies show that circRNAs like circZNF609, circFBXw7, circPiNT exon-2, and circSHPRH regulate tumor growth and act as a tumor promoter by producing functional proteins through template translation (Lin et al., 1986; Yang et al., 2018). CircFBXW7 contains an open reading frame with IRES that facilitates cap-independent translation (Yang et al., 2018). The protein product of circFBXW7 is termed as FBXW7$185 \mathrm{aa}$, which is functionally active, and the overexpression of FBXW7-185aa protein $(21-\mathrm{kDa})$ was found to inhibit tumor progression. In contrast, its knockdown induced a malignant phenotype in vitro and in vivo (Yang et al., 2018). Also, FBXW7185aa reduced c-Myc half-life by altering its stabilization (Yang et al., 2018). In another study, the overexpression of protein SHPRH-146aa encoded by SNF2 histone linker PHD RING helicase (SHPRH) gene was found to reduce the malignant behavior and tumorigenicity of glioblastoma cells (U251 and U373) in vivo and in vitro (Zhang et al., 2018a). Another study showed an 87 amino acid peptide encoded by the long intergenic non-protein-coding RNA p53-induced transcript (LINC-PINT) suppressed glioblastoma cell proliferation in vitro and in vivo (Zhang et al., 2018b). This peptide directly interacts with the PAF1c complex and inhibits many oncogenes' transcriptional elongation, thereby acting as a tumor suppressor (Zhang et al., 2018b). A recent study found that the production of a functional protein (termed as circPPP1R12A-73aa), which has a pro- tumorigenic function in colon cancer, is mediated by the activation of the Hippo-YAP signaling pathway (Zheng X. et al., 2019). Moreover, $\operatorname{circ} \beta$-catenin and circAKT3 produce functional polypeptides $\beta$-catenin-370aa, and AKT3-174aa found to be implicated in tumorigenesis (Liang et al., 2019; Xia et al., 2019). Given the rapid evolution of circRNAs and their protein regulation role, we expect further studies in the next few years on circRNA translation and cancer-associated functional polypeptides.

\section{ROLE OF CircRNAs IN TUMORIGENESIS BY ACTING AS MiRNA SPONGE}

Many studies have highlighted the role of dysregulated circRNA in different diseases, including cancer (Liu et al., 2020). Dysregulation of circRNA occurs in various cancers, which provides a window of opportunity for its development as a viable therapeutic target. Different circRNAs have been reported to influence cellular signaling by binding with micro RNA (miRNA). It has been found that majority of circRNAs act as competitive endogenous RNAs (ceRNAs) and can modulate miRNA activity by binding to miRNA response elements (MREs) (Mitra et al., 2018). Furthermore, the binding of human antigen $\mathrm{R}(\mathrm{HuR})$ with many circRNAs in human cervical carcinoma HPV18 positive HeLa cell lines shows RBP binding sites in circRNAs (Abdelmohsen et al., 2017) and demonstrate the efficacy of circRNAs to scaffold the protein involved in the critical cellular phenomenon.
Similarly, another circRNA, circ-FOXO3, has been found to promote cellular senescence by interacting with ID-1, an anti-senescence protein and a stress pathway related proteins such as HIF1 $\alpha$ and FAK, thus making these proteins unable to exert their functional roles (Du et al., 2017b). CircRNAs have been found to influence oncogenic phenomenon like promoting anchorage independent growth, cell proliferation, angiogenesis, metastasis, and drug resistance in different cancers (Su M. et al., 2019). Since circRNAs are dysregulated in different cancers, it is hypothesized that they can possibly be used as a molecular target as well as diagnostic and prognostic markers in both solid and hematological malignancies (Table 3). In this review, we will further discuss about different circRNAs specifically involved in oncogenesis.

\section{ROLE OF CircRNAs IN SOLID MALIGNANCIES}

\section{Breast Cancer}

Different circRNAs have been explored as diagnostic and prognostic markers in breast cancer (Wang and Fang, 2018). Using a microarray approach, Du et al. (2018) detected increased expression of a circRNA, circ-Dnmt1 in breast cancer in vivo and in vitro. Using different functional assays, they showed that circDNMT1 is involved in cell proliferation and survival by stimulating cellular autophagy (Du et al., 2018). At the molecular level, it was found that circDNMT1 interacts with both p53 and AU-rich element RNA-binding protein 1 (AUF1), thereby promoting their nuclear translocation (Du et al., 2018). In another study by Liang H.F. et al. (2017) circABCB10 was significantly upregulated in breast cancer tissues and was involved in the proliferation and inhibition of apoptosis in breast cancer cells. Furthermore, circABCB1O was found to sponge miR-1271 and promoted carcinogenesis through the circABCB10/miR-1271 axis (Liang H.F. et al., 2017).

$\mathrm{Xu}$ Y. et al. (2018) showed that circ-0005230 was upregulated in breast cancer tissues and found to be associated with adverse phenotypes in patients, thus could be used as a prognostic marker in breast cancer. At the molecular level, circ-0005230 was found to sponge miR-618, which regulated CBX8 expression, increasing cell migratory and invasive capabilities (Xu Y. et al., 2018). Tang H. et al. (2019) did the profiling of circRNAs in Triple-negative breast cancer (TNBC) using microarray and found circKIF4A to be the most upregulated circRNA. Additionally, circKIF4A was also associated with poor survival in TNBC patients (Tang H. et al., 2019). It was also found that circKIF4A promoted cell proliferation and migration by binding to miR-375 and regulated the expression of KIF4A via sponging miR-375 (Tang H. et al., 2019). Another study by $\mathrm{He}$ et al. (2017) found circGFRA1 to be significantly upregulated in TNBC cell lines and tissues. It was also shown that upregulated circGFRA1 correlated with poorer survival in TNBC patients. Moreover, the knockdown of circGFRA1 inhibited proliferation and promoted apoptosis in TNBCs (He et al., 2017). It was subsequently shown that circGFRA1 and GFRA1 regulated their expression by sponging 


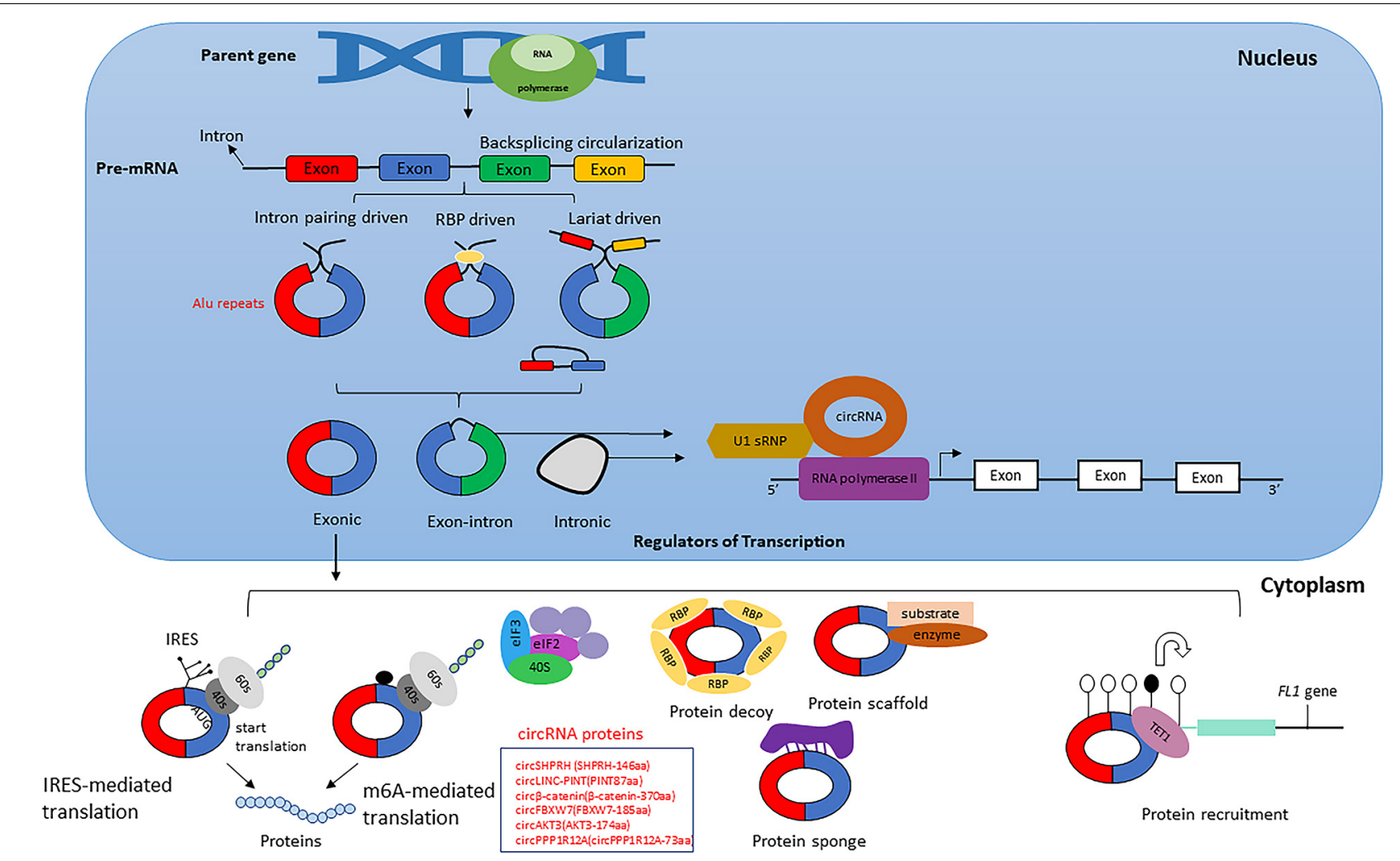

FIGURE 2 | circRNA synthesis. The circRNA is formed by a head-to-tail backsplice junction (BSJ) through a downstream splice donor to an upstream splice acceptor. Chimeric reads align at the BSJ, directly confirming the existence of this non-canonical splice junction. The mates of the chimeric read can align to either of the BSJ forming exons. Supportive reads align to the BSJ forming exons in an orientation that is divergent regarding the direction of transcription but becomes properly inward-facing and convergent for BSJ. Adapted from Ahmed et al. (2016).

miR-34a, thus highlighting the potential of circGFRA1 as a prognostic marker in TNBC (He et al., 2017).

\section{Bladder Cancer}

Zhong et al. (2016) analyzed circRNA profile in bladder cancer using microarray and found that circTCF25 could sequester miR-103a-3p/miR-107, potentially leading to the upregulation of 13 targets related to cell proliferation, migration and invasion. It was demonstrated that the downregulation of miR-103a-3p and miR-107 could increase CDK6 expression and promote proliferation/migration of Bladder cancer cells in vitro and in vivo. This study suggests using circTCF25 as a potential therapeutic target in bladder cancer (Zhong et al., 2016). Zhong et al. (2017) performed a microarray profile of bladder carcinoma and found that circMYLK and VEGFA were significantly upregulated in bladder cancer. At the molecular level, it was found that circMYLK suppressed the activity of miR-29a and regulated the expression of VEGFA, thereby activating the VEGFA/VEGF R2 signaling pathway (Zhong et al., 2017). In vitro data indicated that circMYLK promoted epithelial-mesenchymal transition (EMT), cell proliferation and evasion of apoptosis by activating the Ras/ERK signaling pathway (Zhong et al., 2017). Another study by Su H. et al. (2019) found that the expression of circRNA cTFRC was upregulated and significantly correlated with poor prognosis of bladder cancer patients. Also, cTFRC was found to be a competing endogenous RNA (ceRNA) for miR-107 and mediated TGF- $\beta$-induced EMT in bladder cancer cells ( $\mathrm{Su} \mathrm{H}$. et al., 2019).

Moreover, the downregulation of CTFRC inhibited the invasive potential of bladder cancer cells (Su H. et al., 2019). Indeed, this study highlighted the potential of cTFRC as a prognostic marker in bladder cancer (Su H. et al., 2019). In another study, Li Y. et al. (2019) explored the role of circRNA circMTO1 in bladder cancer. The expression levels of circMTO1 were downregulated in bladder cancer tissues, and reduced circMTO1 levels positively correlated with poor survival in bladder cancer patients. Besides, the overexpression of circMTO1 resulted in the inhibition of EMT, sponging of miR-221 and suppressing the E-cadherin/N-cadherin pathway ( $\mathrm{Li} \mathrm{Y}$. et al., 2019). Overall, the study highlighted the potential of circMTO1 as a prognostic marker and a therapeutic target in bladder cancer (Zhong et al., 2017).

\section{Colorectal Cancer}

Weng et al. (2017) analyzed the clinical significance of ciRS-7, a potential mIR-7 sponge in colorectal cancer (CRC). The study evaluated the effect of ciRS-7 on miR-7, and its target genes EGFR and RAF1. The levels of ciRS-7 were found to be upregulated 
TABLE 3 | CircRNAs as diagnostic and prognostic markers in different cancers and their molecular targets.

\begin{tabular}{|c|c|c|c|c|c|c|}
\hline Cancer type & Circular RNA & Source of sample & Expression in cancer & Molecular targets & Prognostic/Diagnostic potential & References \\
\hline \multirow[t]{3}{*}{ Breast cancer (BC) } & circ-0005230 & Tissues and cell lines & Upregulated & $\begin{array}{l}\text { miR-618 and } \\
\text { CBX8 }\end{array}$ & $\begin{array}{l}\text { Can serve as a prognostic predictor } \\
\text { in } \mathrm{BC} \text { patients }\end{array}$ & Xu Y. et al., 2018 \\
\hline & circKIF4A & Tissues and cell lines & Upregulated & miR-375 & $\begin{array}{l}\text { Can serve as a prognostic } \\
\text { biomarker in TNBC }\end{array}$ & Tang H. et al., 2019 \\
\hline & circGFRA1 & Tissues and cell lines & Upregulated & miR-34a & $\begin{array}{l}\text { Can serve as a diagnostic } \\
\text { biomarker in TNBC }\end{array}$ & He et al., 2017 \\
\hline \multirow[t]{2}{*}{ Bladder cancer } & CTFRC & Tissues & Upregulated & miR-107 & $\begin{array}{l}\text { Can serve as a diagnostic } \\
\text { biomarker in bladder cancer }\end{array}$ & Su H. et al., 2019 \\
\hline & circMTO1 & Tissues & Downregulated & miR-221 & $\begin{array}{l}\text { Can serve as a prognostic } \\
\text { biomarker in bladder cancer }\end{array}$ & Li et al., 2019b \\
\hline \multirow[t]{5}{*}{$\begin{array}{l}\text { Colorectal cancer } \\
\text { (CRC) }\end{array}$} & circVAPA & Plasma & Upregulated & miR-101 & $\begin{array}{l}\text { Can serve as a diagnostic } \\
\text { biomarker in CRC }\end{array}$ & Li X.N. et al., 2019 \\
\hline & $\begin{array}{l}\text { circ RNA panel } \\
\text { (circCCDC66, } \\
\text { circABCC1, } \\
\text { circSTIL) }\end{array}$ & Plasma & Downregulated & - & $\begin{array}{l}\text { Can serve as a diagnostic } \\
\text { biomarker in CRC }\end{array}$ & Lin et al., 2019 \\
\hline & hsa-circ-0004771 & Circulating exosome & Upregulated & - & $\begin{array}{l}\text { Can serve as a diagnostic } \\
\text { biomarker in CRC }\end{array}$ & Pan et al., 2019 \\
\hline & circlTGA7 & Tissues and cell lines & Downregulated & miR-370-3p & $\begin{array}{l}\text { Can serve as a diagnostic } \\
\text { biomarker in CRC }\end{array}$ & Li et al., 2018h \\
\hline & circCCDC66 & Tissue & Upregulated & - & $\begin{array}{l}\text { Can serve as a diagnostic } \\
\text { biomarker in CRC }\end{array}$ & Hsiao et al., 2017 \\
\hline \multirow[t]{5}{*}{ Gastric cancer (GC) } & hsa-circ-0014717 & Tissues and gastric juice & Downregulated & - & $\begin{array}{l}\text { Can serve as a diagnostic } \\
\text { biomarker in GC }\end{array}$ & Shao et al., 2017 \\
\hline & hsa-circ-00000520 & Tissues, plasma and cell lines & Downregulated & $\begin{array}{l}\text { Nine miRNAs and nine candidates } \\
\text { mRNA that are predicted to have } \\
\text { interaction with hsa_circ_0000520 }\end{array}$ & $\begin{array}{l}\text { Can serve as a diagnostic } \\
\text { biomarker in GC }\end{array}$ & Sun et al., 2018 \\
\hline & hsa-circ-0000096 & Tissues and cell lines & Downregulated & cyclin D1, CDK6, MMP-2 and MMP-9 & $\begin{array}{l}\text { Can serve as a diagnostic } \\
\text { biomarker in GC }\end{array}$ & Lidonnici et al., 2017 \\
\hline & circNRIP1 & Tissues and cell lines & Upregulated & miR-149-5p & $\begin{array}{l}\text { Can serve both as a diagnostic and } \\
\text { prognostic biomarker in GC }\end{array}$ & Zhang X. et al., 2019 \\
\hline & circPVT1 & Tissues & Upregulated & miR-125 & $\begin{array}{l}\text { Can serve as a prognostic } \\
\text { biomarker in GC }\end{array}$ & Chen J. et al., 2017 \\
\hline $\begin{array}{l}\text { Hepatocellular } \\
\text { carcinoma (HCC) }\end{array}$ & circSMARCA5 & Tissues and plasma samples & Downregulated & - & $\begin{array}{l}\text { Can serve as a prognostic } \\
\text { biomarker in HCC }\end{array}$ & LiY. et al., 2019 \\
\hline
\end{tabular}


TABLE 3 | Continued

\section{hsa-circ-0075930}

Lung cancer (LC) CircFARSA

hsa-circ-0013958

CiRS-7

circPRMT5

circPVT1

hsa-circ-0081001

\section{Source of sample}

Tissues

Tissues, cell lines and serum

Plasma

Tissues

Tissues

Tissues

Tissues

Tissues and plasma

Cell lines and tissue

Tissues, plasma and cell line

Tissues

Tissues and cell lines

Tissues, serum, and cell lines

Cell lines, tissues and serum

\section{Expression in cancer}

Upregulated

Upregulated

Upregulated

Downregulated

Upregulated

Upregulated

Upregulated

Upregulated

Upregulated

Upregulated

Upregulated

Upregulated

Upregulated

Upregulated
miR-484

\section{Molecular targets}

miR-892a and miR-328-3p

miR-582-3p

$-$

Twist1, Vimentin

RBM3

miR-200a

miR-330-5p and miR-326

miR-134

Promoted cell proliferation and growth

miR-377, miR-382 and miR-498

$\mathrm{ABCB} 1$

\section{Prognostic/Diagnostic potential Reference}

Can serve as a diagnostic

biomarker in HCC

Can serve as a diagnostic

biomarker in HCC

Can serve as a diagnostic Yu et al., 2020

biomarker in $\mathrm{HCC}$

can serve as a biomarker for

evaluating the tumor burden of

Qiu et al., 2019

HCC patients

Can serve as a diagnostic

biomarker in HCC

Can serve as a prognostic

biomarker in $\mathrm{HCC}$

Can serve as a prognostic

biomarker in $\mathrm{HCC}$

Can serve as a diagnostic

biomarker in NSCLC 2

Can serve as a prognostic

biomarker in NSCLC

Can serve as a diagnostic

biomarker in $\mathrm{LAC}^{3}$

Can serve as a prognostic

biomarker in NSCLC

Can serve as a diagnostic

biomarker in NSCLC

Can serve as a diagnostic

biomarker in OS

Wei et al., 2020

Zhang et al., 2018d

Can serve both as a diagnostic and prognostic biomarker in OS 


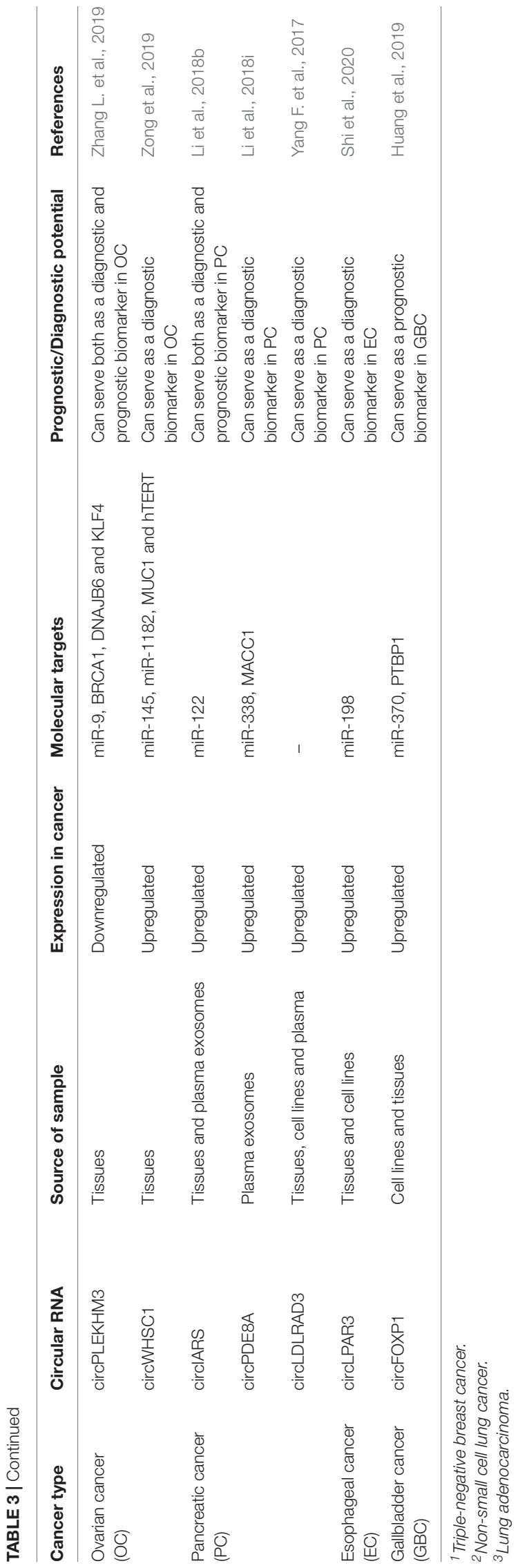

in CRC tissues as compared to normal mucosae. Moreover, the increased expression of ciRS-7 in CRC cell lines (HCT116 and HT29) led to the blocking of miR-7 tumor-suppressive effects and an aggressive oncogenic phenotype (Weng et al., 2017). This study highlighted the efficacy of ciRS-7 as a promising prognostic biomarker and a potential therapeutic target in CRC patients (Weng et al., 2017). Another study by Zeng et al. (2018) found that circHIPK3 was significantly upregulated in CRC tissues and cell lines and positively correlated with metastasis and advanced clinical stage in CRC patients. Molecular analysis indicated that attenuation of circHIPK3 inhibited cell proliferation, migration, invasion, and induced cell death in CRC cell lines (Zeng et al., 2018).

Moreover, circHIPK3 was found to sponge tumor suppressor miR-7, and the overexpression of circHIPK 3 effectively reversed the inhibition of CRC cell proliferation induced by miR-7, thereby demonstrating the tumor-promoting role of circHIPK3 in CRC (Zeng et al., 2018). Furthermore, the silencing of circHIPK3, combined with miR-7 overexpression resulted in cell proliferation inhibition in CRC xenograft animal models (Zeng et al., 2018). Overall, the study indicated the tremendous potential of circHIPK3 as a prognostic marker and therapeutic target in CRC (Zeng et al., 2018). A recent study by Zhang et al. (2018c) found hsa_circ_0007534 to be significantly upregulated in CRC tumor tissues and correlated with tumor stage and lymph node metastasis. Moreover, the silencing of hsa_circ_0007534 resulted in apoptosis and reduced proliferation in CRC cell lines (Zhang et al., 2018c). Another study showed the upregulation of circRNA hsa-circ-0020397 and the downregulation of miR-138 in CRC cells (Zhang X.L. et al., 2017). Furthermore, hsa-circ0020397 was found to antagonize the activity of miR-138 by influencing its target genes like telomerase reverse transcriptase (TERT) and programmed death-ligand 1 (PD-L1) leading to increased cell proliferation and invasion in CRC (Zhang X.L. et al., 2017).

Many circRNAs in CRC have been shown to have diagnostic potential [reviewed in Hao et al. (2019)]. In a study by Li X.N. et al. (2019) circVAPA was found to be upregulated in the tissues and plasma of CRC and promoted CRC progression by sponging miR-101. CircVAPA promotes CRC cell proliferation, migration, invasion, and inhibit apoptosis in CRC cell lines (Li X.N. et al., 2019). Another study by Lin et al. (2019) evaluated the efficacy of using plasma circRNAs panel (circCCDC66, circABCC1 and circSTIL) as diagnostic markers in CRC. The plasma levels of the three circRNAs were found to be significantly reduced in CRC patients compared to healthy controls (Lin et al., 2019). Specifically, circCCDC66 and circSTIL were found to be efficient early-stage diagnostic markers in CRC (Lin et al., 2019).

Moreover, it was shown that combining the three circRNA panel with carcinoembryonic antigen (CEA) and carbohydrate antigen 19-9 (CA19-9) might improve CRC diagnosis (Lin et al., 2019). A study by Pan et al. (2019) found that exosomal circRNA hsa-circ-0004771 to be significantly upregulated in CRC patients' sera. Moreover, the elevated expression of exosomal hsa-circ0004771 in the serum of CRC patients was found to be tumorderived and showed a high diagnostic value in CRC. Another study by Li et al. (2018h) found that circRNA circITGA7 and 
its host gene ITGA7 were downregulated in CRC tissues and cell lines. Also, circITGA7 was found to negatively regulate the proliferative Ras signaling pathway by binding to miR-370-3p and antagonizing its suppression of neurofibromin 1, a negative regulator of the Ras signaling pathway (Li et al., 2018h). Another study showed the upregulation and association of circCCDC66 with poor CRC prognosis (Hsiao et al., 2017). circCCDC66 was found to influence cell proliferation, migration, invasion, and anchorage-independent growth in CRC cells, demonstrating its potential as a CRC biomarker (Hsiao et al., 2017).

\section{Esophageal Squamous Cell Carcinoma}

A study by Xia et al. (2016) found that hsa-circ-0067934 was overexpressed in Esophageal squamous cell carcinoma (ESCC) tissues. Additionally, high expression of hsa-circ0067934 associated with poor differentiation, I-II T stage, and I-II TNM stage and promoted ESCC proliferation and migration. Moreover, the silencing of hsa-circ-0067934 by siRNA resulted in the inhibition of proliferation, migration, and blocking of the cell cycle in ESCC cells, thereby suggesting its potential as a biomarker and therapeutic target in ESCC (Xia et al., 2016). Another study found that circRNA, circLPAR3, to be highly expressed in ESCC tissues, upregulated MET gene expression by sponging miR-198 and promoted the migration, invasion and metastasis of ESCC cells through the activation of RAS/MAPK and PI3K/Akt signaling pathways, thus showing its potential as a diagnostic target in ESCC (Shi et al., 2020).

\section{Gastric Cancer}

A study by Pan $H$. et al. (2018) highlighted the clinical significance of ciRS-7 in gastric cancer (GC) and found that the overexpression of ciRS-7 blocked miR-7-induced tumor suppression in GC cell lines and induced an aggressive phenotype by antagonizing PTEN/PI3K/AKT pathway. This study indicates the possibility of using cIRS-7 as a therapeutic target and a prognostic biomarker in GC (Pan H. et al., 2018). Another study reported circRNA-0023642 to be upregulated in GC tissues and cell lines (Zhou et al., 2018). The altered expression of circRNA0023642 influenced the EMT pathway and its associated genes like N-cadherin, vimentin, snail, and E-cadherin. Moreover, the downregulation of circRNA-0023642 suppressed proliferation, migration, and invasion of GC cells, thereby suggesting circRNA0023642 as a potential therapeutic target in GC (Zhou et al., 2018). Shao et al. (2017) did a global circRNA expression profile using microarray, and hsa-circ-0014717 was the most downregulated circRNA in GC. In addition, the expression levels of hsa-circ-0014717 were found to be associated with tumor stage, distal metastasis, tissue carcinoembryonic antigen (CEA) and carbohydrate antigen 19-9 (CA19-9) expression (Shao et al., 2017). Similarly, another circRNA hsa-circ-0000520 was found to be downregulated in GC tissues and plasma (Sun et al., 2018). It was further seen that the hsa-circ-0000520 level in GC tissues negatively associated with the TNM stage (Sun et al., 2018). Another study by Li et al. highlighted the diagnostic utility of circRNA hsa-circ-0000096, which was found to be downregulated in GC tissues and GC cell lines (Lidonnici et al., 2017). Moreover, the levels of cyclin D1, cyclin-dependent kinase 6 (CDK6), matrix metalloproteinases (MMPs) such as MMP-2 and MMP-9 were found to be significantly reduced both in vitro and in vivo in GC. Furthermore, the knockdown of hsa-circ-0000096 was shown to inhibit the proliferation and migration of GC cells in vivo and in vitro (Lidonnici et al., 2017). Another study by Zhang X. et al. (2019) found the expression of circNRIP1 to be significantly upregulated in GC. They found that knockdown of circNRIP1 blocked proliferation, migration, invasion, and the expression of AKT1 by sponging miR-149-5p and promoting oncogenesis in GC cells (Zhang X. et al., 2019). Thus, the inhibition of circNRIP1 can serve as a promising therapeutic target in GC (Zhang X. et al., 2019). Chen J. et al. (2017) identified a new circRNA circPVT1 that was found to be upregulated in GC tissues. CircPVT1 was found to sponge members of the miR125 family and served as an independent prognostic marker for overall survival (OS) and disease-free survival (DFS) in GC (Li and Huang, 2017).

\section{Glioma}

Li et al. (2018a) found hsa-circ-0046701 to be significantly upregulated in glioma tissue and cell lines. Moreover, hsacirc-0046701 was found to sponge miR-142-3p and regulated the expression of Integrin alpha-V beta-8 (ITGB8) (Li et al., 2018a). A negative correlation was found between hsa-circ0046701 and miR-142-3p expressions, indicating the role of hsa_circ_0046701/miR-142-3p/ITGB8 axis in the oncogenesis of glioma ( $\mathrm{Li}$ et al., 2018a). Xu H. et al. (2018) studied the relationship between circNFIX and miR-34a-5p using CIRCexplorer2, circRNA-finder, CIRI, find-circ, and MapSplice2 in glioma. The study found circNFIX to be upregulated in glioma tissues and acted as a sponge for miR-34a-5p, thereby regulating its interaction with $\mathrm{NOTCH} 1$ (Xu H. et al., 2018). Besides, an increased level of circNFIX promoted cell propagation and migration in glioma (Xu $\mathrm{H}$. et al., 2018). An in vitro study found that silencing of circRNA, cZNF292 suppressed the proliferative and angiogenic potential of glioma cells. It was also found that cZNF292 resulted in cell cycle arrest at the S/G2/M phase with the downregulation of PRR11, Cyclin A, p-CDK2, VEGFR-1/2, p-VEGFR-1/2, and EGFR, proteins involved in cell cycle progression, thus implicating its role as a potential therapeutic target in glioma (Yang et al., 2016).

\section{Gall Bladder Cancer}

Kai et al. (2018) studied the role of circHIPK3 in gall bladder cancer (GBC) and found elevated expression of circHIPK3 in GBC cell lines. The silencing of circHIPK3 inhibited the proliferation of gall bladder cell lines (Kai et al., 2018). Moreover, circHIPK3 sponged the tumor-suppressive miR-124 leading to an increased expression of miR-124 targets, including rho-associated protein kinase 1 (ROCK1) and CDK6, thereby showing the potential of circHIPK3 as a potential diagnostic marker and therapeutic target in GBC (Kai et al., 2018). Another study by Wang et al. found that the upregulation of circFOXP1 promoted cell proliferation, migration, and GBC invasion. Moreover, the study showed that circFOXP1 promoted the Warburg effect in GBC by interacting with polypyrimidine tract binding protein 
1 (PTBP1) and promoting the expression of pyruvate kinase, liver, and RBC (PKLR). Additionally, the study also found that circFOXP1 promoted the Warburg effect in GBC by acting as a sponge of miR-370 that regulates the expression of PKLR, thus showing the potential of circFOXP1 as a prognostic biomarker in GBC (Wang S. et al., 2019). An interesting study by Huang et al. (2019) found that the increased expression of circERBB2 promoted GBC progression by regulating the nuclear localization of proliferation-associated protein 2G4 (PA2G4) and modulating ribosomal DNA transcription.

\section{Hepatocellular Carcinoma}

Guan et al. (2018) studied circRNAs profile in hepatocellular carcinoma (HCC) using human circular RNA microarray and showed that hsa-circ-0016788 was found to be upregulated in both HCC tissues and cell lines. hsa-circ-0016788 was found to promote the proliferation and invasion of HCC through the hsa-circ-0016788/miR-486/CDK4 pathway (Guan et al., 2018). Another study by Meng et al. (2018) reported that Twist1 regulates vimentin expression by upregulating Cullin 2 circRNA (circ10720), which absorbs miRNAs that target vimentin. Thus, the Culllin2 circRNA based mechanism involved in the Twist1mediated regulation of vimentin during the process of EMT serves as a potential therapeutic target for the treatment of HCC (Meng et al., 2018). Another study found the expression of circSMARCA5 to be downregulated in HCC tissues and plasma samples (Li et al., 2019b). The overexpression of circSMARCA5 was found to inhibit proliferation, invasion, and increase apoptosis in HCC cells. Furthermore, circSMARCA5 correlated with tumor differentiation, TNM stage, cancer invasion, and cancer diameter in HCC (Li et al., 2019b). Wei et al. (2020) found circCDYL (chromodomain Y like) to be upregulated in HCC. CircCDYL was found to interact with mRNAs encoding hepatoma-derived growth factor (HDGF) and hypoxia-inducible factor asparagine hydroxylase (HIF1AN) by sponging miR892a and miR-328-3p and influencing their downstream target (Wei et al., 2020). Furthermore, the authors demonstrated that circCDYL expression, combined with HDGF and HIF1AN, can function as independent markers for discrimination of early staged HCC (Wei et al., 2020). Thus, circCDYL provides a possibility for the early treatment of HCC (Wei et al., 2020). Zhang et al. (2018d) explored the diagnostic role of circ-104075, which was found to be highly upregulated in HCC tissues, cell lines, and serum. Besides, the expression of circ-104075 was found to be positively regulated by the hepatocyte nuclear factor 4 alpha (HNF4A), and circ-104075 acted as a ceRNA to upregulate YAP expression by sponging miR-582-3p (Zhang et al., 2018d). Yu et al. (2020) explored the diagnostic potential of hepatitis B virus (HBV) related HCC and plasma circRNAs. The study showed that plasma circRNA panel (circPanel) containing three circRNAs (hsa-circ-0000976, hsa-circ-0007750 and hsacirc-0139897) could accurately detect Small-HCC, AFP-negative HCC, and AFP-negative Small-HCC (Yu et al., 2020). Qiu et al. (2019) found circADAMTS13, derived from Exon 13-14 of the ADAMTS13 gene, to be downregulated in HCC tumor tissues. circADAMTS13 is negatively associated with tumor size but positively associated with prognosis in HCC (Qiu et al.,
2019). circADAMTS13 interacted with miR-484 and served as a tumor suppressor during HCC progression by sponging miR-484 (Qiu et al., 2019). Dong et al. (2019) found that SCD-circRNA2 was significantly upregulated in HCC. SCDcircRNA2 was found to be regulated by RNA-binding protein 3 (RBM3), indicative of a short recurrence-free survival and poor overall survival in HCC patients (Dong et al., 2019). Another study by Li et al. (2018e) found that circ-101368 was upregulated in HCC tissue samples and the overexpression of circ-101368 correlated with poor prognosis in HCC. At the molecular level, knockdown of circ-101368 suppressed the migration and protein levels of high mobility group box 1 (HMGB1), receptor for advanced glycation end products (RAGE) and nuclear factor kappa-light-chain-enhancer of activated $B$ $(\mathrm{NF}-\kappa \mathrm{B})$ while increasing the E-cadherin expression in HCC (Li et al., 2018e).

\section{Lung Cancer}

Zhang et al. (2018e) studied the expression of CDR1as, which is found to sponge miRNA-7 (miR-7). It was found that CDR1as levels increased with the development of non-small cell lung cancer (NSCLC) and negatively correlated with the expression of mIR-7 (Zhang et al., 2018e). Patients with high expression of CDR1 were found to exhibit high TNM stage, increased lymph node metastasis (LNM) and reduced overall survival (OS) (Zhang et al., 2018e). CDR1as was found to function as a miR-7 sponge to upregulate the target genes of miR-7 like EGFR, cyclin E1 (CCNE1), and phosphatidylinositol-4,5bisphosphate 3-kinase catalytic subunit delta (PIK3CD) (Zhang et al., 2018e). Another circRNA, circ-0067934, was upregulated in NSCLC tissues and cell lines and its expression was significantly associated with TNM stage, lymph node status, and distant metastasis (Wang and Li, 2018). The study suggested that circ-0067934 is an EMT marker as it regulates EMT genes such as vimentin, $\mathrm{N}$-cadherin, and E-cadherin (Wang and Li, 2018). Another study by Zhou et al. (2019) demonstrated the regulatory mechanism of circENO1 on its host gene enolase 1 (ENO1) and its role in glycolysis and tumor progression. The expression of both circENO1 and its host gene ENO1 was found to be upregulated in lung adenocarcinoma (LAC) cells. Mechanistically, the upregulated expression of ENO1 was due to the interaction of circENO1 as a ceRNA with miR-22-3p (Zhou et al., 2019). Moreover, the silencing of circENO1 resulted in the retardation of glycolysis and suppressed proliferation, invasion and EMT in LAC cells (Zhou et al., 2019). Dong et al. found that circFARSA, a circRNA derived from exon 5-7 of the FARSA gene, was found to be upregulated in NSCLC patients (Hang et al., 2018). In vitro experiments using NSCLC cell lines indicated that overexpression of circFARSA promoted cell migration and invasion (Hang et al., 2018). Moreover, in silico studies suggested that circFARSA might sponge miR-330-5p and miR-326 and relieve their inhibitory effects on oncogene fatty acid synthase (Hang et al., 2018). The expression of another circRNA, hsacirc-0075930 was found to be upregulated in NSCLC cell lines and tissues (Li et al., 2018d). In vitro studies indicated that the depletion of hsa-circ-0075930 inhibited cell proliferation and induced cell cycle arrest and reversed EMT in NSCLC cells 
(Li et al., 2018d). Another study by Zhu et al. (2017) found hsacirc-0013958 to be upregulated in LAC and associated with TNM stage and lymphatic metastasis. In addition, hsa-circ-0013958 was found to sponge miR-134, thereby upregulating the expression of cyclin D1, which has an oncogenic role in the development of lung cancer (Zhu et al., 2017). In a retrospective study, it was found that circRNA, ciRS-7 expression was elevated in NSCLC tissue and positively correlated with tumor size, lymph node metastasis, and TNM stage (Yan et al., 2018). Inhibition of ciRS-7 reduced proliferation and promoted cellular apoptosis in A549 lung cancer cell lines (Yan et al., 2018). Wang L. et al. (2018) studied the regulation and function of circRNA, hsacirc-0008305 (circPTK2) in TGF- $\beta$-induced EMT and tumor metastasis in NSCLC. It was found that circPTK2 and TIF1 $\gamma$ were significantly downregulated in NSCLC cells undergoing EMT induced by TGF- $\beta$. CircPTK2 was found to function as a sponge of miR-429/miR-200b-3p, and miR-429/miR-200b-3p and influenced their downstream targets (Wang L. et al., 2018). Another circRNA, circPRMT5 was found to be highly expressed in NSCLC tissues and cell lines and positively correlated with large tumor size, advanced clinical stage, lymph node metastasis as well as poor prognosis (Wang Y. et al., 2019). At the molecular level, circPRMT5 simultaneously sponged three miRNAs (miR377, miR-382, and miR-498) and alleviated their repression on the oncogenic enhancer of zeste homolog 2 (EZH2), thereby facilitating NSCLC progression (Wang Y. et al., 2019).

\section{Osteosarcoma}

Many circular RNAs have been studied in osteosarcoma (OS) (Wan et al., 2020). circ-0016347 was found to act as a positive regulator of proliferation and invasion in OS cells, and it was identified as a sponge of miR-214 (Jin et al., 2017). The expression of another circRNA, circUBAP2, was found to be significantly increased in human OS tissues. circUBAP2 was found to promote osteosarcoma cell growth and inhibit apoptosis by inhibiting the expression of miR-143 and its downstream target B Cell Lymphoma 2 (Bcl-2) (Zhang H. et al., 2017). Another circRNA, circ-0009910, was overexpressed in OS and involved in the downregulation of miR-449a (Deng et al., 2018). Mechanistic studies have shown circ-0009910/miR-449a/IL6R axis to be a regulator of JAK1/STAT3 signaling pathway and a promoter of oncogenesis in osteosarcoma (Deng et al., 2018). Kun-Peng et al. (2018b) explored the role of circPVT1 in osteosarcoma and found that circPVT1 was significantly upregulated in OS. The knockdown of circPVT1 weakens the resistance to doxorubicin and cisplatin in OS cells by decreasing the expression of drug resistance gene ATP binding cassette subfamily $B$ member 1 (ABCB1) (Kun-Peng et al., 2018b). Another circRNA, hsacirc-0081001, was found to be significantly upregulated in the OS cell lines, tissues, serums and associated with poor overall survival and therefore demonstrated the potential of hsa-circ0081001 as a potential prognostic or diagnostic biomarker for OS (Kun-Peng et al., 2018a).

\section{Ovarian Cancer}

Chen Q. et al. (2018) found the expression of hsa-circ-0061140 to be upregulated in ovarian cancer (OC) cell lines. The knockdown of hsa-circ-0061140 was found to suppress cell viability and proliferation of OC. Mechanistically, hsa-circ-0061140 sponged miR-370 and suppressed the expression of FOXM1 by acting as a ceRNA of miR-370, thus promoting oncogenesis in OC (Chen Q. et al., 2018). Another circular RNA, circPLEKHM3 was found to be downregulated in OC tissues and peritoneal metastatic OC compared to primary ovarian carcinomas (Zhang X. et al., 2019). CircPLEKHM3 was found to function as a tumor suppressor in OC by sponging miR-9 to regulate the endogenous expression of breast cancer 1 (BRCA1), DNA heat shock protein family (Hsp40) member B6 (DNAJB6) and kruppel like factor 4 (KLF4) which consequently inactivates oncogenic AKT1 signaling (Zhang L. et al., 2019). Another study found the expression of circular RNA circWHSC1 to be upregulated in OC tissues (Zong et al., 2019). Mechanistically, circWHSC1 increased cell proliferation, migration and invasion, and inhibited cell apoptosis in OC (Zong et al., 2019). circWHSC1 sponges miR-145 and miR-1182 and upregulates the expression of downstream targets mucin 1 (MUC1) and human telomerase reverse transcriptase (hTERT) (Zong et al., 2019).

\section{Pancreatic Cancer}

Li et al. (2018b) explored the role of circIARS in pancreatic ductal adenocarcinoma (PDAC) and found the expression of circIARS to be upregulated in the tissues and exosomes of the PDAC patients. CircIARS negatively correlated with miR-122 and ZO-1 and positively correlated with RhoA and RhoA-GTP levels, increased F-actin expression and focal adhesion, thereby promoting tumor invasion and metastasis, which suggests the role of circIARS as a prognostic marker in PDAC (Li et al., 2018b). Another circRNA, circPDE8A, was found to promote the invasive growth of PDAC cells by upregulating mesenchymal-epithelial transition (MET) and acted as a ceRNA for miR-338 to regulate metastasis-associated in colon cancer protein 1 (MACC1) and stimulate invasive growth of PDAC via the MACC/MET/ERK or AKT pathway (Li et al., 2018i). Furthermore, it was found that exosomal circPDE8A was associated with prognosis and disease progression in PDAC patients (Li et al., 2018i). Yang F. et al. (2017) found circLDLRAD3 to be upregulated in pancreatic cancer in addition to the significant association with venous invasion, lymphatic invasion, and metastasis.

\section{ROLE OF CircRNAs IN HAEMATOLOGICAL MALIGNANCIES}

\section{Acute Myeloid Leukemia}

Many circRNAs are associated with resistance in acute myeloid leukemia (AML) (Wu Z. et al., 2019). Shang et al. (2019) studied the role of circRNAs mediated chemoresistance in AML. Their study found that circPAN3 is upregulated in refractory and recurrent $\mathrm{AML}$ patient tissues and doxorubicin (ADM)-resistant THP-1 AML cell lines compared to nontransformed tissue and THP-1 AML cell lines (Shang et al., 2019). Mechanistically, circPAN3 decreased the expression of X-linked apoptosis protein inhibitor (XIAP) and further suggested that circPAN3 could be a crucial mediator of chemoresistance in 
AML cells by exploiting the circPAN3-miR-153-5p / miR-1835p-XIAP axis (Shang et al., 2019). Another study by Yi et al. (2019) explored circvimentin (VIM) expression in AML patients and found it to be significantly upregulated in AML and correlated with WBC count. The study showed that circVIM expression could serve as a biomarker in differentiating AML patients from controls (Yi et al., 2019). Also, survival analyses in AML patients showed that over-expressed circVIM associated with shorter OS and leukemia-free survival (LFS) in wholecohort AML, non-acute promyelocytic leukemia AML and cytogenetically normal-AML patients (Yi et al., 2019). Another study by Chen H. et al. (2018) found circANAPC7 to be most significantly upregulated in AML patients. The study predicted that circANAPC7 acts as a sponge for the miR-181 family and is associated with oncogenic pathways, thus demonstrating the potential of circANAPC7 as a potential biomarker for AML (Chen H. et al., 2018). Dong et al. explored the oncogenic potential of circular RNA DLEU2 (circDLEU2), microRNA 496 (miR-496), and Protein Kinase CAMP-activated catalytic subunit beta (PRKACB) in AML cell lines (Wu et al., 2018). CircDLEU2 was upregulated in AML tissues and promoted AML cell proliferation and inhibited cell apoptosis in vivo ( $\mathrm{Wu}$ et al., 2018). Moreover, circDLEU2 was found to inhibit miR496 expression and promote PRKACB expression. This study collectively indicated that circDLEU2 accelerated human AML by suppressing miR-496 and promoting PRKACB expression (Wu et al., 2018).

\section{Chronic Myeloid Leukemia}

BCR-ABL1 is a fusion protein kinase derived from a reciprocal translocation between chromosomes nine and 22 and is a crucial protein for Chronic Myeloid Leukemia (CML) pathogenesis (Pan Y. et al., 2018). Tyrosine kinase inhibitors (TKIs) against BCR-ABL1 have revolutionized CML therapy; however, 25\% of CML patients will switch TKIs at least once during their lifetime due to TKI intolerance or resistance (Bower et al., 2016), which is generally associated with mutations in the kinase domain (KD) of BCR-ABL1. Pan Y. et al. (2018) found that a novel circRNA named circBA9.3, derived from BCR-ABL1, can efficiently promote the proliferation and inhibit apoptosis of CML cells. Moreover, TKI resistance was associated with elevated circBA9.3 expression and positively correlated with the level of BCR-ABL1 (Pan Y. et al., 2018). Furthermore, the augmentation of cytoplasmic-ABL1 (c-ABL) and BCR-ABL1 oncoprotein expression by circBA9.3 suggests that circBA9.3 might serve as a therapeutic target in CML patients showing TKI resistance (Pan Y. et al., 2018). Another study by Liu et al. (2018) constructed an hsa-circ-0080145-mediated competing endogenous RNA (ceRNA) regulatory network. They found that hsa-circ- 0080145 was found to regulate CML cell proliferation by sponging miR-29b.

\section{CircRNAs AS BIOMARKERS}

Numerous studies have demonstrated that circRNAs has emerging potential as a clinically relevant and disease-specific molecular biomarker in cancer and other complex diseases. Growing evidence suggests that circRNAs can be considered as promising biomarkers for the early diagnosis, metastasis, prognosis, and drug resistance of tumors due to their stable structure, the long half-life, tissue specificity, and abundance, and their presence in body fluids (Guo et al., 2014; Chen and Yang, 2015; You et al., 2015; Enuka et al., 2016; Yang Y. et al., 2017). CircRNAs are considered useful tools for the early detection of solid tumors, for example, hsa_circ_0043265 could be used as a biomarker for the early diagnosis of NSCLC due to its low expression in the early stages (Ren et al., 2020). The abundance of circMYLK can be useful as an early diagnosis tool and treatment of liver cancer (Li et al., 2019a).

Moreover, circRNAs play a key role in tumor metastasis, Yang et al. (2020) found that the higher expression of circPTK2 can be positively correlated with a high survival rate in colorectal cancer patients. These findings suggest that circPTK2 may be a therapeutic target for metastatic colorectal cancer and a promising prognostic biomarker for early diagnosis. In PDAC, a high CircBFAR expression level was positively correlated with the tumor-node-metastasis (TNM) stage and was associated with poor prognosis. Hence, circBFAR could be used as a prognostic marker and therapeutic target for PDAC (Guo et al., 2020). Besides, many circular RNAs can be used as markers for drug resistance also. hsa_circ_0006528 (Gao et al., 2017), circMTO1 (Chen M. et al., 2019), circ_0001546 (Wu et al., 2020), and circ-LARP4 (Hu et al., 2020) exhibit abnormal expression levels in drug-resistant cells, suggesting that they could be used as diagnostic markers for drug resistance in tumors. CircRNAs have become accepted as biomarkers for multi-stage tumors in different cancer types. Thus far, many studies have demonstrated the potential of circRNAs as promising cancer biomarkers. The abnormal (upregulated or downregulated) expression levels of different circRNAs in various cancers are depicted in Figure 3. Recently studies have shown that circRNAs are also potential diagnostic markers for neurodegenerative diseases (Table 4). Several studies have indicated that circular RNAs are involved in repair and recovery after stroke, highlighting their potential therapeutic importance in regulating biological processes in brain injury due to ischemic stroke. Bai et al. (2018) demonstrated a critical role for circular RNA DLGAP4 (circDLGAP4) in repairing ischemic stroke brain damage. The gain of function of circDLGAP4 significantly reduced neurological deficits and decreased infarct areas and blood-brain barrier damage in a mouse stroke model. The study suggests that circDLGAP4 may serve as a novel therapeutic target for acute ischemic injury.

Furthermore, a recently identified circRNA, circHECTD1 can directly bind to MIR142, resulting in astrocyte activation and contributing to cerebral infarction. Silencing of circHECTD1 expression can ameliorate cerebral infarction by inhibiting astrocyte activation (Han et al., 2018). These studies indicate that circHECTD1 and circDLGAP4 could be envisioned as a novel biomarker and therapeutic target for stroke.

If circRNA detection methods can be effectively applied in clinical practice, they have the great potential to serve as 


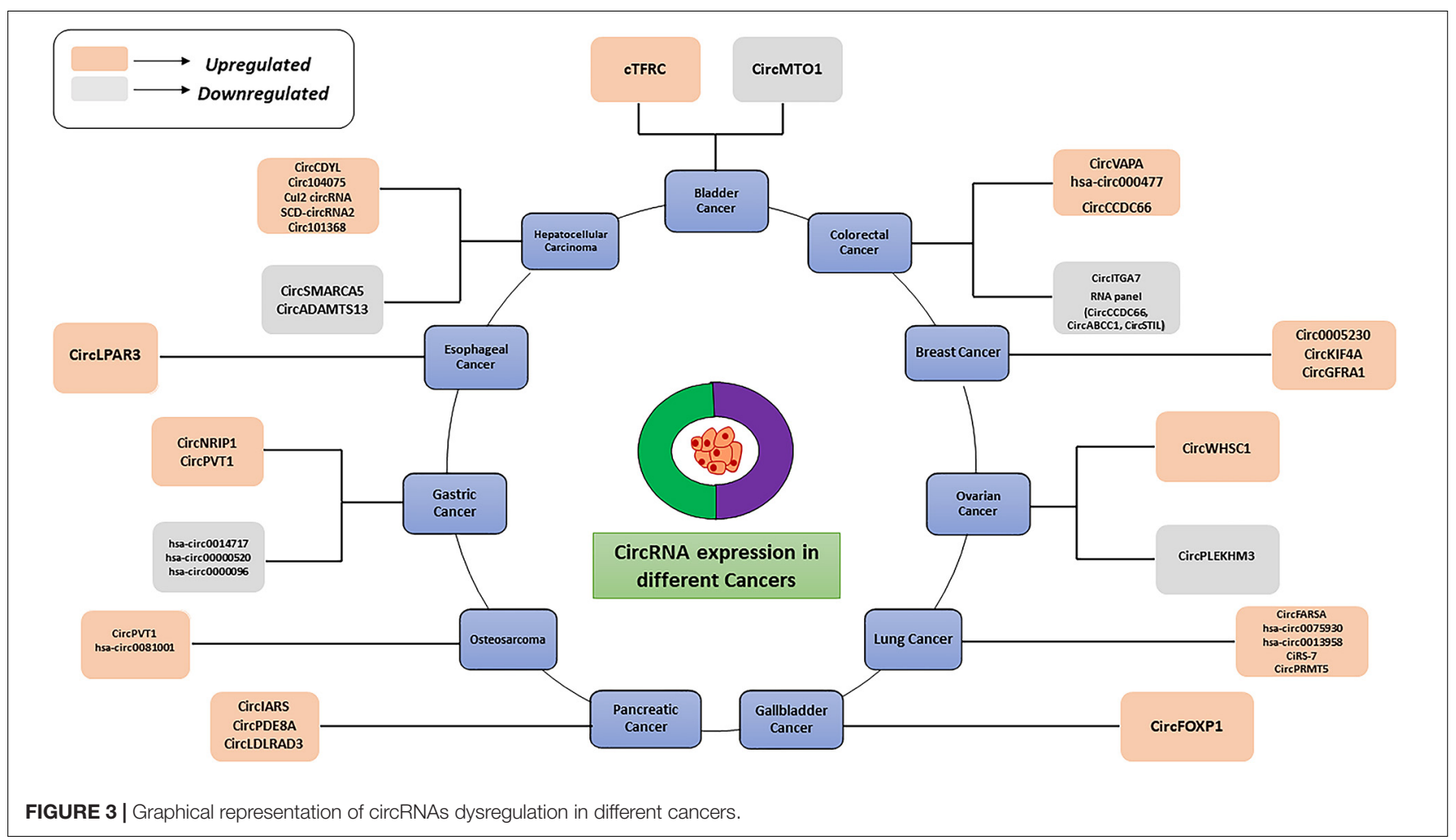

TABLE 4 | CircRNAs as biomarkers in neurodegenerative diseases.

\begin{tabular}{|c|c|c|c|}
\hline Disease type & Circular RNA & Expression level & References \\
\hline \multirow[t]{3}{*}{ Temporal lobe epilepsy } & circ-EFCAB2 & Upregulated & Li et al., 2018c \\
\hline & circ-DROSHA & Downregulated & Li et al., 2018c \\
\hline & circRNA-0067835 & Downregulated & Gong et al., 2018 \\
\hline \multirow[t]{2}{*}{ Multiple sclerosis } & circ_0005402, circ_0035560 & Downregulated & Iparraguirre et al., 2017 \\
\hline & hsa_circ_0106803 & Downregulated & Cardamone et al., 2017 \\
\hline \multirow[t]{2}{*}{ Alzheimer's disease } & CDR1as/ ciRS-7 & Downregulated & Akhter, 2018 \\
\hline & circPVT1 & Downregulated & Panda et al., 2017 \\
\hline \multirow[t]{2}{*}{ Parkinson's disease } & CDR1as/ciRS-7 & Upregulated & Kumar et al., 2017 \\
\hline & circzip-2 & Downregulated & Kumar et al., 2018 \\
\hline \multirow[t]{3}{*}{ Ischemic stroke } & circPDS5B & Upregulated & Zuo et al., 2020 \\
\hline & circCDC14A & Upregulated & Zuo et al., 2020 \\
\hline & circHECTD1 & Upregulated & Han et al., 2018 \\
\hline
\end{tabular}

early diagnostic markers to avoid patient distress in many lifethreatening diseases.

\section{CircRNAs AS THERAPEUTIC TARGETS}

High throughput deep RNA-sequencing followed by advanced bioinformatic analysis and functional characterization leads to discovering novel circRNAs with clinical significance. Researchers identified many circRNAs are related to cancer's clinicopathological features, such as tumor metastasis, epithelial to mesenchymal transition, tumor stemness, and recurrence. Many studies have confirmed that circRNAs play a vital role in cancer initiation, progression, sensitivity to therapy, cancer stemness, and drug resistance. Many of the circRNAs identified can be used as a potential drug target in cancer, mainly due to their involvement in inducing therapy resistance via sponging miRNAs and alerting oncogenic signaling mechanisms. Numerous experiments have demonstrated that circRNAs play a regulatory role in drug resistance in many cancer types, and they can be used as potential drug targets. In lung cancer, CircPVT1 was found to mediate drug resistance via the miR145-5p/ABCC1 axis. CircPVT1 knockout sensitizes tumor cells to conventional chemotherapeutic agents like cisplatin and pemetrexed (Zheng and Xu, 2020). This indicates that abrogating circRNAs in tumors can sensitize them to drugs effectively. Additionally, overexpression of circESRP1 and inhibition of the TGF- $\beta$ signaling pathway can regulate the tumor response to 
chemotherapy in lung cancer (Huang W. et al., 2020). Circular RNAs viz., circ_0002483, and Circ_0076305 are involved in drug sensitivity in lung cancer via binding the target genes ( $\mathrm{Li}$ X. et al., 2019). In breast cancer, regulating Hsa_circ_0006528, circKDM4C, and circMTO1 can effectively sensitize drug resistance in cancer types (Gao et al., 2017; Ma et al., 2019). In GC, CircPVT1 acts as a carcinogenic factor by mediating paclitaxel resistance by upregulating via ZEB1/ miR-124-3p axis (Liu Y.Y. et al., 2019). CircFN1 regulates GC cell apoptosis through sponging miR-182-5p and promotes cisplatin resistance in GC, suggesting that circFN1 could be a potential therapeutic target (Huang X.X. et al., 2020). CircAKT3 and circCCDC66 are also involved in cisplatin resistance by altering target signaling pathways (Xu G. et al., 2020). In GC cells, apatinib resistance is mediated through circRACGAP1 via circRACGAP1/miR3657/ATG7 axis (Ma et al., 2020). In colorectal cancer, circular RNA circ_0000338 has a tumor-suppressive effect and can enhance colorectal cancer cells' chemosensitivity (Hon et al., 2019). Besides, a circular RNA, CIRS-122 (Wang et al., 2020) hsa_circ_0048234, was also involved in drug resistance in CRC (Xiong et al., 2017). Novel circular RNAs viz., hsa_circ_0000285, and circCdrlas, sensitize bladder cancer cells to cisplatin by restoring the expression of target genes (Chi et al., 2019; Yuan et al., 2019). In ovarian cancer, circCdrlas reduces cisplatin resistance by inhibiting miR-1270 (Zhao Z. et al., 2019). CircCELSR1 (hsa_circ_0063809) is upregulated in paclitaxelresistant ovarian cancer tissues and cells (Zhang et al., 2020). Silencing of circCELSR1 may enhance the cytotoxic effect of paclitaxel in ovarian cancer cells suggesting circRNAs as promising therapeutic targets in many cancer types.

Surprisingly, many circRNAs exhibit an immunomodulatory function, as they are actively involved in immune regulation and autoimmune pathway regulation. Studies revealed that cells can recognize endogenous circRNA and in vitro synthesized circRNA by retinoic acid-inducible gene I (RIG-I), which can activate the autoimmune pathway (Chen Y.G. et al., 2017). It has been proved that cells can distinguish between endogenous circRNAs and exogenous or in vitro synthesized circRNAs by identifying the presence of N6-Methyladenosine modification (m6A) (Chen Y.G. et al., 2019). They further concluded that exogenous circRNAs without m6A modification could alter gene expression in the autoimmune pathway. Moreover, a recent study shows circRNAs can competitively bind double-stranded RNA-activated protein kinase (PKR) to extensively regulate cellular immune signaling pathways (Liu Y.Y. et al., 2019). Liu et al. (2016) identified differentially regulated circRNA, chondrocyte extracellular matrix-related circRNA (circRNACER) in osteoarthritis (OA), which can modulate the process of cartilage extracellular matrix injury via IL- $1 \beta$ and TNF- $\alpha$ regulation. This indicates that circRNA-CER may be a potential therapeutic target in osteoarthritis (OA) (Liu et al., 2016). Many studies indicate that circRNAs are implicated in Systemic lupus erythematosus (SLE), a chronic and incurable autoimmune disease. circRNAs are probably vital factors in SLE due to their functions as miRNA sponges. Wang X. et al. (2018) reported the downregulation of circIBTK in SLE, and they further revealed that circIBTK served as a miR-29b sponge to inhibit DNA demethylation and AKT signaling. Moreover, they have experimentally proved that the artificial overexpression of circRNAs can help reduce PKR activity in PBMCs in SLE patients, which is beneficial for treating SLE and other autoimmune diseases (Liu C.X. et al., 2019).

Many researchers confirmed that circRNAs play roles in bone marrow failure and hematologic malignancy. Xia $\mathrm{P}$. et al. (2018) experimentally proved that circRNA cia-aGAS controls the balance between self-renewal and differentiation of hematopoietic stem cells (HSCs), and its deficiency disrupts the host homeostasis, leading to bone marrow failure and hematologic malignancy. This evidence suggests that circRNAs are involved in tumor stemness and can act as a possible therapeutic target.

Circular RNAs have also been shown to play vital roles in virus infections, providing a new strategy for developing vaccines against viruses, especially RNA viruses. The transfection of purified circRNAs generated in vitro into mammalian cells can induce the expression of innate immunity genes and thus protect against viral infection. This suggests that cells can distinguish endogenous and foreign circRNAs, dependent on the intron encoding the circRNAs (Chen Y.G. et al., 2017). In another study, Li et al. (2017) found that the antiviral function of circular RNA is universal and is mediated through NF90/NF110 release. Generally, the expression of circRNAs decreases during viral infection, so NF90/NF110 is released from circRNPs and binds to viral mRNAs to play an antiviral role.

Circular RNAs are extraordinarily enriched in the mammalian brain, and they are more abundant than their corresponding linear transcripts or parental mRNAs (Rybak-Wolf et al., 2015). Notably, circRNAs expressed in brain tissues have been reproducibly detected in human peripheral blood samples as they can pass through the blood-brain barrier (Rybak-Wolf et al., 2015). These findings suggest that the blood-borne circRNAs may be potential diagnostic biomarkers and reveal the pathophysiology for ischemic stroke and other brain-related diseases (Lu et al., 2020). Many circRNAs are upregulated in the mammalian brain during neuronal differentiation, indicating circRNAs are potentially involved in neurological disorders (Rybak-Wolf et al., 2015). CDRlas is an endogenous circRNA that is highly expressed in the human brain, acting as a miR-7 sponge, thus plays a key role in regulating Parkinson's disease. Studies revealed that MiR-7 can modulate alphasynuclein expression, a protein that always accumulates at the onset of Parkinson's disease (Junn et al., 2009). This evidence strongly suggests that CDRlas may be a therapeutic target in Parkinson's disease.

Additionally, CDR1as is implicated in Alzheimer's disease by binding to miR-7, thereby reducing the ability of miR-7 to regulate ubiquitin-conjugating enzyme E2 A (UBE2A), a protein that decreases rapidly in Alzheimer's disease (AD) and other neurological diseases (Akhter, 2018). Moreover, CDRlas inhibits the translation of NF-k $\beta$, indirectly impairs protein functions that underlie AD's development (Shi et al., 2017). As evidenced by the above studies and our conceptual understanding, circRNAs can be considered a potential biomarker and therapeutic target in $\mathrm{AD}$ diagnosis and treatment. Additionally, CDR1as is also 
implicated in cardiovascular diseases, mostly associated with myocardial infarction. Furthermore, CDRlas induces myocardial infarction by sponging mir7, protecting cardiac pericardium (Taibi et al., 2014). Another novel circRNA, mitochondrial fission and apoptosis-related circRNA (MFACR), sponges miR652-3p-MTP18 to mediate cardiomyocyte apoptosis, leads to mitochondrial fission, and ultimately promotes the development of myocardial infarction (Wang K. et al., 2017). Recent studies and current evidence indicate that circRNAs play a crucial role in controlling cellular dynamics in many types of tissue; thus, circRNAs may serve as a potential therapeutic avenue for many complex diseases, although further research is required for clinical applications to be feasible.

\section{CONCLUSION}

Over the last few decades, the significance of circRNAs in numerous pathological processes has gradually been realized; while initially known as splicing redundant byproducts, clinical studies have managed to uncover the hidden characteristics and ability of circRNAs to serve as ideal biomarkers in different pathologies. Unlike other RNA species, circRNAs have prognostic or diagnostic value due to high stability and specific cancer expression. Understanding the molecular mechanisms/regulatory roles of circRNAs in various transcriptional and translational processes in the eukaryotic machinery is essential to elucidate their human diseases' roles, most importantly, cancer. Apart from the notorious role of circRNAs as miRNA sponges, further studies are required to reveal other mechanisms manifested by circRNAs. Although the expression of circRNAs has been extensively studied, their function remains inconclusive in normal physiological processes and human diseases. Many mechanistic aspects of how circRNAs potentially work in various cancers by affecting oncogenic pathways need to be better clarified, and we are still a long way from their development as a therapeutic or prognostic target that warrants thorough analysis of their mechanism

\section{REFERENCES}

Abdelmohsen, K., Panda, A. C., Munk, R., Grammatikakis, I., Dudekula, D. B., De, S., et al. (2017). Identification of HuR target circular RNAs uncovers suppression of PABPN1 translation by CircPABPN1. RNA Biol. 14, 361-369. doi: 10.1080/15476286.2017.1279788

Abe, N., Hiroshima, M., Maruyama, H., Nakashima, Y., Nakano, Y., Matsuda, A., et al. (2013). Rolling circle amplification in a prokaryotic translation system using small circular RNA. Angew. Chem. Int. Ed. Engl. 52, 7004-7008. doi: 10.1002/anie. 201302044

Abe, N., Matsumoto, K., Nishihara, M., Nakano, Y., Shibata, A., Maruyama, H., et al. (2015). Rolling circle translation of circular RNA in living human cells. Sci. Rep. 5:16435.

Ahmed, I., Karedath, T., Al-Dasim, F. M., and Malek, J. A. (2019). Identification of human genetic variants controlling circular RNA expression. RNA 25, 17651778. doi: 10.1261/rna.071654.119

Ahmed, I., Karedath, T., Andrews, S. S., Al-Azwani, I. K., Mohamoud, Y. A., Querleu, D., et al. (2016). Altered expression pattern of circular RNAs in primary and metastatic sites of epithelial ovarian carcinoma. Oncotarget 7 , 36366-36381. doi: 10.18632/oncotarget.8917 of action. Research in this area must step away from simple quantification and therapeutic inference into mechanistic studies with a translational benefit. Developing technologies that enable sufficient single-cell level identification and modulation of circRNAs without disrupting their linear RNA partners may help gain more insight into the regulatory functions of circRNAs and thus help enhance the production of strategies that target the circRNA network in various human diseases. As circRNAs are generated from pre-mRNA back splicing, different bioinformatics algorithms have been designed to characterize circRNAs using different annotations to read back splicing sites. It will always make sense to develop the algorithm based on specific circRNA to avoid false reads and correlate the results using independent analysis by different platforms.

\section{AUTHOR CONTRIBUTIONS}

$\mathrm{SN}, \mathrm{AB}, \mathrm{MS}$, IA, and TK: collected the literature, wrote the manuscript, and generated the figures. AR, SH, PB, RR, FJ, SU, GC, DB, WE-R, and MF: critical revision and editing of the scientific contents. AB, IA, TK, MM, and MH: conceived and designed the review contents and contributed to the manuscript writing and editing. All authors read and approved the final manuscript.

\section{FUNDING}

$\mathrm{MH}$ is funded by a grant (5071012001) from Sidra Medicine Doha, Qatar. AB is supported by Sidra Medicine internal grant (5011041002). This work was further supported by the Ramanujan Fellowship grant from the Science and Engineering Research Board, Government of India (SB/S2/RJN029/2019) to IA and by Ramalingaswami Fellowship (Grant Number: D.O.NO.BT/HRD/35/02/2006) from the Department of Biotechnology, Government of India, New Delhi to MM.

Akers, N. K., Schadt, E. E., and Losic, B. (2018). STAR chimeric post for rapid detection of circular RNA and fusion transcripts. Bioinformatics 34, 2364-2370. doi: 10.1093/bioinformatics/bty091

Akhter, R. (2018). Circular RNA and Alzheimer's disease. Adv. Exp. Med. Biol. 1087, 239-243.

Andrés-León, E., Núñez-Torres, R., and Rojas, A. M. (2016). miARma-Seq: a comprehensive tool for miRNA, mRNA and circRNA analysis. Sci. Rep. 6:25749.

Ashwal-Fluss, R., Meyer, M., Pamudurti, N. R., Ivanov, A., Bartok, O., Hanan, M., et al. (2014). circRNA biogenesis competes with pre-mRNA splicing. Mol. Cell 56, 55-66. doi: 10.1016/j.molcel.2014.08.019

Bai, Y., Zhang, Y., Han, B., Yang, L., Chen, X., Huang, R., et al. (2018). Circular RNA DLGAP4 ameliorates ischemic stroke outcomes by targeting miR-143 to regulate endothelial-mesenchymal transition associated with blood-brain barrier Integrity. J. Neurosci. 38, 32-50. doi: 10.1523/jneurosci.1348-17.2017

Bartsch, D., Zirkel, A., and Kurian, L. (2018). Characterization of circular RNAs (circRNA) associated with the translation machinery. Methods Mol. Biol. 1724, 159-166. doi: 10.1007/978-1-4939-7562-4_13

Bentley, D. L. (2014). Coupling mRNA processing with transcription in time and space. Nat. Rev. Genet. 15, 163-175. doi: 10.1038/nrg3662 
Bower, H., Björkholm, M., Dickman, P. W., Höglund, M., Lambert, P. C., and Andersson, T. M. (2016). Life expectancy of patients with chronic myeloid leukemia approaches the life expectancy of the general population. J. Clin. Oncol. 34, 2851-2857. doi: 10.1200/jco.2015.66.2866

Capel, B., Swain, A., Nicolis, S., Hacker, A., Walter, M., Koopman, P., et al. (1993). Circular transcripts of the testis-determining gene Sry in adult mouse testis. Cell 73, 1019-1030. doi: 10.1016/0092-8674(93)90279-y

Cardamone, G., Paraboschi, E. M., Rimoldi, V., Duga, S., Soldà, G., and Asselta, R. (2017). The characterization of GSDMB splicing and backsplicing profiles identifies novel Isoforms and a circular RNA that are dysregulated in multiple sclerosis. Int. J. Mol. Sci. 18:576. doi: 10.3390/ijms18030576

Chen, C. Y., and Chuang, T. J. (2019). NCLcomparator: systematically postscreening non-co-linear transcripts (circular, trans-spliced, or fusion RNAs) identified from various detectors. BMC Bioinform. 20:3. doi: 10.1186/s12859018-2589-0

Chen, D.-F., Zhang, L.-J., Tan, K., and Jing, Q. (2018). Application of droplet digital PCR in quantitative detection of the cell-free circulating circRNAs. Biotechnol. Biotechnol. Equ. 32, 116-123. doi: 10.1080/13102818.2017.1398596

Chen, H., Liu, T., Liu, J., Feng, Y., Wang, B., Wang, J., et al. (2018). Circ-ANAPC7 is upregulated in acute myeloid leukemia and appears to target the MiR-181 family. Cell Physiol. Biochem. 47, 1998-2007. doi: 10.1159/000491468

Chen, N., Zhao, G., Yan, X., Lv, Z., Yin, H., Zhang, S., et al. (2018). A novel FLI1 exonic circular RNA promotes metastasis in breast cancer by coordinately regulating TET1 and DNMT1. Genome Biol. 19:218.

Chen, Q., Zhang, J., He, Y., and Wang, Y. (2018). hsa_circ_0061140 Knockdown reverses FOXM1-mediated cell growth and metastasis in ovarian cancer through miR-370 sponge activity. Mol. Ther. Nucleic Acids 13, 55-63. doi: 10.1016/j.omtn.2018.08.010

Chen, X., Chen, R. X., Wei, W. S., Li, Y. H., Feng, Z. H., Tan, L., et al. (2018). PRMT5 circular RNA promotes metastasis of urothelial carcinoma of the bladder through sponging miR-30c to induce epithelial-mesenchymal transition. Clin. Cancer Res. 24, 6319-6330. doi: 10.1158/1078-0432.ccr-181270

Chen, J., Li, Y., Zheng, Q., Bao, C., He, J., Chen, B., et al. (2017). Circular RNA profile identifies circPVT1 as a proliferative factor and prognostic marker in gastric cancer. Cancer Lett. 388, 208-219. doi: 10.1016/j.canlet.2016.12.006

Chen, Y. G., Kim, M. V., Chen, X., Batista, P. J., Aoyama, S., Wilusz, J. E., et al. (2017). Sensing self and foreign circular RNAs by intron identity. Mol. Cell 67, 228-238.e225.

Chen, L. L., and Yang, L. (2015). Regulation of circRNA biogenesis. RNA Biol. 12, 381-388. doi: 10.1080/15476286.2015.1020271

Chen, M., Ai, G., Zhou, J., Mao, W., Li, H., and Guo, J. (2019). circMTO1 promotes tumorigenesis and chemoresistance of cervical cancer via regulating miR-6893. Biomed. Pharmacother. 117:109064. doi: 10.1016/j.biopha.2019.109064

Chen, Y. G., Chen, R., Ahmad, S., Verma, R., Kasturi, S. P., Amaya, L., et al. (2019). N6-Methyladenosine modification controls circular RNA immunity. Mol. Cell 76, 96-109.e109.

Chen, X., Han, P., Zhou, T., Guo, X., Song, X., and Li, Y. (2016). circRNADb: a comprehensive database for human circular RNAs with protein-coding annotations. Sci. Rep. 6:34985.

Chi, B. J., Zhao, D. M., Liu, L., Yin, X. Z., Wang, F. F., Bi, S., et al. (2019). Downregulation of hsa_circ_0000285 serves as a prognostic biomarker for bladder cancer and is involved in cisplatin resistance. Neoplasma 66, 197-202. doi: 10.4149/neo_2018_180318n185

Chuang, T. J., Wu, C. S., Chen, C. Y., Hung, L. Y., Chiang, T. W., and Yang, M. Y. (2016). NCLscan: accurate identification of non-co-linear transcripts (fusion, trans-splicing and circular RNA) with a good balance between sensitivity and precision. Nucleic Acids Res. 44:e29. doi: 10.1093/nar/gkv1013

Conn, S. J., Pillman, K. A., Toubia, J., Conn, V. M., Salmanidis, M., Phillips, C. A., et al. (2015). The RNA binding protein quaking regulates formation of circRNAs. Cell 160, 1125-1134. doi: 10.1016/j.cell.2015.02.014

Cortés-López, M., Gruner, M. R., Cooper, D. A., Gruner, H. N., Voda, A. I., Van Der Linden, A. M., et al. (2018). Global accumulation of circRNAs during aging in Caenorhabditis elegans. BMC Genom. 19:8. doi: 10.1186/s12864-017-4386-y

Dahl, M., Daugaard, I., Andersen, M. S., Hansen, T. B., Grønbæk, K., Kjems, J., et al. (2018). Enzyme-free digital counting of endogenous circular RNA molecules in B-cell malignancies. Lab. Invest. 98, 1657-1669. doi: 10.1038/s41374-0180108-6
Deng, N., Li, L., Gao, J., Zhou, J., Wang, Y., Wang, C., et al. (2018). Hsa_circ_0009910 promotes carcinogenesis by promoting the expression of miR-449a target IL6R in osteosarcoma. Biochem. Biophys. Res. Commun. 495, 189-196. doi: 10.1016/j.bbrc.2017.11.028

Dobin, A., Davis, C. A., Schlesinger, F., Drenkow, J., Zaleski, C., Jha, S., et al. (2013). STAR: ultrafast universal RNA-seq aligner. Bioinformatics 29, 15-21. doi: 10.1093/bioinformatics/bts635

Dong, R., Zhang, X.-O., Zhang, Y., Ma, X.-K., Chen, L.-L., and Yang, L. (2016). CircRNA-derived pseudogenes. Cell Res. 26, 747-750. doi: 10.1038/cr.2016.42

Dong, W., Dai, Z. H., Liu, F. C., Guo, X. G., Ge, C. M., Ding, J., et al. (2019). The RNA-binding protein RBM3 promotes cell proliferation in hepatocellular carcinoma by regulating circular RNA SCD-circRNA 2 production. eBio Med. 45, 155-167. doi: 10.1016/j.ebiom.2019.06.030

Dou, Y., Cha, D. J., Franklin, J. L., Higginbotham, J. N., Jeppesen, D. K., Weaver, A. M., et al. (2016). Circular RNAs are down-regulated in KRAS mutant colon cancer cells and can be transferred to exosomes. Sci. Rep. 6:37982.

Du, W. W., Fang, L., Yang, W., Wu, N., Awan, F. M., Yang, Z., et al. (2017a). Induction of tumor apoptosis through a circular RNA enhancing Foxo3 activity. Cell Death Differ. 24, 357-370. doi: 10.1038/cdd.2016.133

Du, W. W., Yang, W., Chen, Y., Wu, Z. K., Foster, F. S., Yang, Z., et al. (2017b). Foxo3 circular RNA promotes cardiac senescence by modulating multiple factors associated with stress and senescence responses. Eur. Heart J. 38, 1402-1412.

Du, W. W., Yang, W., Li, X., Awan, F. M., Yang, Z., Fang, L., et al. (2018). A circular RNA circ-DNMT1 enhances breast cancer progression by activating autophagy. Oncogene 37, 5829-5842. doi: 10.1038/s41388-018-0369-y

Enuka, Y., Lauriola, M., Feldman, M. E., Sas-Chen, A., Ulitsky, I., and Yarden, Y. (2016). Circular RNAs are long-lived and display only minimal early alterations in response to a growth factor. Nucleic Acids Res. 44, 1370-1383. doi: 10.1093/ nar/gkv1367

Errichelli, L., Dini Modigliani, S., Laneve, P., Colantoni, A., Legnini, I., Capauto, D., et al. (2017). FUS affects circular RNA expression in murine embryonic stem cell-derived motor neurons. Nat. Commun. 8:14741.

Ferreira, H. J., Davalos, V., De Moura, M. C., Soler, M., Perez-Salvia, M., Bueno-Costa, A., et al. (2018). Circular RNA CpG island hypermethylationassociated silencing in human cancer. Oncotarget 9, 29208-29219. doi: 10. 18632/oncotarget. 25673

Ferrero, G., Licheri, N., Coscujuela Tarrero, L., De Intinis, C., Miano, V., Calogero, R. A., et al. (2019). Docker4Circ: a framework for the reproducible characterization of circRNAs from RNA-Seq Data. Int. J. Mol. Sci. 21:293. doi: 10.3390/ijms 21010293

Gaffo, E., Bonizzato, A., Kronnie, G. T., and Bortoluzzi, S. (2017). CirComPara: a multi-method comparative bioinformatics pipeline to detect and study circRNAs from RNA-seq data. Noncod. RNA 3:8. doi: 10.3390/ncrna3010008

Gao, D., Zhang, X., Liu, B., Meng, D., Fang, K., Guo, Z., et al. (2017). Screening circular RNA related to chemotherapeutic resistance in breast cancer. Epigenomics 9, 1175-1188. doi: 10.2217/epi-2017-0055

Gao, Y., Wang, H., Zhang, H., Wang, Y., Chen, J., and Gu, L. (2018). PRAPI: post-transcriptional regulation analysis pipeline for Iso-Seq. Bioinformatics 34, 1580-1582. doi: 10.1093/bioinformatics/btx830

Ghosal, S., Das, S., Sen, R., Basak, P., and Chakrabarti, J. (2013). Circ2Traits: a comprehensive database for circular RNA potentially associated with disease and traits. Front. Genet. 4:283. doi: 10.3389/fgene.2013.00283

Glažar, P., Papavasileiou, P., and Rajewsky, N. (2014). circBase: a database for circular RNAs. RNA 20, 1666-1670. doi: 10.1261/rna.0436 87.113

Gong, G. H., An, F. M., Wang, Y., Bian, M., Wang, D., and Wei, C. X. (2018). Comprehensive circular RNA profiling reveals the regulatory role of the CircRNA-0067835/miR-155 pathway in temporal lobe epilepsy. Cell Physiol. Biochem. 51, 1399-1409. doi: 10.1159/000495589

Guan, Z., Tan, J., Gao, W., Li, X., Yang, Y., Li, X., et al. (2018). Circular RNA hsa_circ_0016788 regulates hepatocellular carcinoma tumorigenesis through miR-486/CDK4 pathway. J. Cell Physiol. 234, 500-508. doi: 10.1002/jcp.26612

Guo, J. U., Agarwal, V., Guo, H., and Bartel, D. P. (2014). Expanded identification and characterization of mammalian circular RNAs. Genome Biol. 15:409.

Guo, X., Zhou, Q., Su, D., Luo, Y., Fu, Z., Huang, L., et al. (2020). Circular RNA circBFAR promotes the progression of pancreatic ductal adenocarcinoma via the miR-34b-5p/MET/Akt axis. Mol. Cancer 19:83. 
Hammond, S. M., Boettcher, S., Caudy, A. A., Kobayashi, R., and Hannon, G. J. (2001). Argonaute2, a link between genetic and biochemical analyses of RNAi. Science 293, 1146-1150. doi: 10.1126/science.1064023

Han, B., Zhang, Y., Zhang, Y., Bai, Y., Chen, X., Huang, R., et al. (2018). Novel insight into circular RNA HECTD1 in astrocyte activation via autophagy by targeting MIR142-TIPARP: implications for cerebral ischemic stroke. Autophagy 14, 1164-1184. doi: 10.1080/15548627.2018.1458173

Han, D., Li, J., Wang, H., Su, X., Hou, J., Gu, Y., et al. (2017). Circular RNA circMTO1 acts as the sponge of microRNA-9 to suppress hepatocellular carcinoma progression. Hepatology 66, 1151-1164. doi: 10.1002/hep.29270

Hang, D., Zhou, J., Qin, N., Zhou, W., Ma, H., Jin, G., et al. (2018). A novel plasma circular RNA circFARSA is a potential biomarker for non-small cell lung cancer. Cancer Med. 7, 2783-2791. doi: 10.1002/cam4.1514

Hansen, T. B. (2018). Improved circRNA identification by combining prediction algorithms. Front. Cell Dev. Biol. 6:20. doi: 10.3389/fcell.2018.00020

Hansen, T. B., Jensen, T. I., Clausen, B. H., Bramsen, J. B., Finsen, B., Damgaard, C. K., et al. (2013). Natural RNA circles function as efficient microRNA sponges. Nature 495, 384-388. doi: 10.1038/nature11993

Hao, S., Cong, L., Qu, R., Liu, R., Zhang, G., and Li, Y. (2019). Emerging roles of circular RNAs in colorectal cancer. Oncotargets Ther. 12, 4765-4777. doi: 10.2147/ott.s208235

He, R., Liu, P., Xie, X., Zhou, Y., Liao, Q., Xiong, W., et al. (2017). circGFRA1 and GFRA1 act as ceRNAs in triple negative breast cancer by regulating miR-34a. J. Exp. Clin. Cancer Res. 36:145.

Hoffmann, S., Otto, C., Doose, G., Tanzer, A., Langenberger, D., Christ, S., et al. (2014). A multi-split mapping algorithm for circular RNA, splicing, transsplicing and fusion detection. Genome Biol. 15:R34.

Holdt, L. M., Stahringer, A., Sass, K., Pichler, G., Kulak, N. A., Wilfert, W., et al. (2016). Circular non-coding RNA ANRIL modulates ribosomal RNA maturation and atherosclerosis in humans. Nat. Commun. 7:12429.

Hon, K. W., Ab-Mutalib, N. S., Abdullah, N. M. A., Jamal, R., and Abu, N. (2019). Extracellular vesicle-derived circular RNAs confers chemoresistance in colorectal cancer. Sci. Rep. 9:16497.

Hsiao, K. Y., Lin, Y. C., Gupta, S. K., Chang, N., Yen, L., Sun, H. S., et al. (2017). Noncoding effects of circular RNA CCDC66 promote colon cancer growth and metastasis. Cancer Res. 77, 2339-2350. doi: 10.1158/0008-5472.can-161883

Hsu, M. T., and Coca-Prados, M. (1979). Electron microscopic evidence for the circular form of RNA in the cytoplasm of eukaryotic cells. Nature 280, 339-340. doi: 10.1038/280339a0

Hu, Y., Gu, J., Shen, H., Shao, T., Li, S., Wang, W., et al. (2020). Circular RNA LARP4 correlates with decreased Enneking stage, better histological response, and prolonged survival profiles, and it elevates chemosensitivity to cisplatin and doxorubicin via sponging microRNA-424 in osteosarcoma. J. Clin. Lab. Anal. 34:e23045.

Huang, A., Zheng, H., Wu, Z., Chen, M., and Huang, Y. (2020). Circular RNAprotein interactions: functions, mechanisms, and identification. Theranostics 10, 3503-3517. doi: 10.7150/thno.42174

Huang, W., Yang, Y., Wu, J., Niu, Y., Yao, Y., Zhang, J., et al. (2020). Circular RNA cESRP1 sensitises small cell lung cancer cells to chemotherapy by sponging miR-93-5p to inhibit TGF- $\beta$ signalling. Cell Death Differ. 27, 1709-1727. doi: 10.1038/s41418-019-0455-x

Huang, X. X., Zhang, Q., Hu, H., Jin, Y., Zeng, A. L., Xia, Y. B., et al. (2020). A novel circular RNA circFN1 enhances cisplatin resistance in gastric cancer via sponging miR-182-5p. J. Cell Biochem.

Huang, C., Liang, D., Tatomer, D. C., and Wilusz, J. E. (2018). A length-dependent evolutionarily conserved pathway controls nuclear export of circular RNAs. Genes Dev. 32, 639-644. doi: 10.1101/gad.314856.118

Huang, X., He, M., Huang, S., Lin, R., Zhan, M., Yang, D., et al. (2019). Circular RNA circERBB2 promotes gallbladder cancer progression by regulating PA2G4dependent rDNA transcription. Mol. Cancer 18:166.

Humphreys, D. T., Fossat, N., Demuth, M., Tam, P. P. L., and Ho, J. W. K. (2019). Ularcirc: visualization and enhanced analysis of circular RNAs via back and canonical forward splicing. Nucleic Acids Res. 47:e123. doi: 10.1093/nar/gkz718

Iparraguirre, L., Muñoz-Culla, M., Prada-Luengo, I., Castillo-Triviño, T., Olascoaga, J., and Otaegui, D. (2017). Circular RNA profiling reveals that circular RNAs from ANXA2 can be used as new biomarkers for multiple sclerosis. Hum. Mol. Genet. 26, 3564-3572. doi: 10.1093/hmg/ddx243
Ivanov, A., Memczak, S., Wyler, E., Torti, F., Porath, H. T., Orejuela, M. R., et al. (2015). Analysis of intron sequences reveals hallmarks of circular RNA biogenesis in animals. Cell Rep. 10, 170-177. doi: 10.1016/j.celrep.2014.12.019

Izuogu, O. G., Alhasan, A. A., Alafghani, H. M., Santibanez-Koref, M., Elliott, D. J., and Jackson, M. S. (2016). PTESFinder: a computational method to identify post-transcriptional exon shuffling (PTES) events. BMC Bioinform. 17:31. doi: 10.1186/s12859-016-0881-4

Jakobi, T., and Dieterich, C. (2018). Deep computational circular RNA analytics from RNA-seq data. Methods Mol. Biol. 1724, 9-25. doi: 10.1007/978-1-49397562-4_2

Jakobi, T., and Dieterich, C. (2019). Computational approaches for circular RNA analysis. Wiley Interdiscip. Rev. RNA 10:e1528. doi: 10.1002/wrna.1528

Jakobi, T., Uvarovskii, A., and Dieterich, C. (2019). circtools-a one-stop software solution for circular RNA research. Bioinformatics 35, 2326-2328. doi: 10.1093/ bioinformatics/bty 948

Jeck, W. R., Sorrentino, J. A., Wang, K., Slevin, M. K., Burd, C. E., Liu, J., et al. (2013). Circular RNAs are abundant, conserved, and associated with ALU repeats. RNA 19, 141-157. doi: 10.1261/rna.035667.112

Jia, G. Y., Wang, D. L., Xue, M. Z., Liu, Y. W., Pei, Y. C., Yang, Y. Q., et al. (2019). CircRNAFisher: a systematic computational approach for de novo circular RNA identification. Acta Pharmacol. Sin. 40, 55-63. doi: 10.1038/s41401-018-0063-1

Jin, H., Jin, X., Zhang, H., and Wang, W. (2017). Circular RNA hsa-circ0016347 promotes proliferation, invasion and metastasis of osteosarcoma cells. Oncotarget 8, 25571-25581. doi: 10.18632/oncotarget.16104

Junn, E., Lee, K. W., Jeong, B. S., Chan, T. W., Im, J. Y., and Mouradian, M. M. (2009). Repression of alpha-synuclein expression and toxicity by microRNA-7. Proc. Natl. Acad. Sci. U.S.A. 106, 13052-13057. doi: 10.1073/pnas.0906277106

Kai, D., Yannian, L., Yitian, C., Dinghao, G., Xin, Z., and Wu, J. (2018). Circular RNA HIPK3 promotes gallbladder cancer cell growth by sponging microRNA124. Biochem. Biophys. Res. Commun. 503, 863-869. doi: 10.1016/j.bbrc.2018. 06.088

Karedath, T., Ahmed, I., Al Ameri, W., Al-Dasim, F. M., Andrews, S. S., Samuel, S., et al. (2019). Silencing of ANKRD12 circRNA induces molecular and functional changes associated with invasive phenotypes. BMC Cancer 19:565. doi: 10.1186/ s12885-019-5723-0

Kelly, S., Greenman, C., Cook, P. R., and Papantonis, A. (2015). Exon skipping is correlated with exon circularization. J. Mol. Biol. 427, 2414-2417. doi: 10.1016/ j.jmb.2015.02.018

Kim, D., Pertea, G., Trapnell, C., Pimentel, H., Kelley, R., and Salzberg, S. L. (2013). TopHat2: accurate alignment of transcriptomes in the presence of insertions, deletions and gene fusions. Genome Biol. 14:R36.

Kleaveland, B., Shi, C. Y., Stefano, J., and Bartel, D. P. (2018). A network of noncoding regulatory RNAs acts in the mammalian brain. Cell 174, 350362.e317.

Kramer, M. C., Liang, D., Tatomer, D. C., Gold, B., March, Z. M., Cherry, S., et al. (2015). Combinatorial control of Drosophila circular RNA expression by intronic repeats, hnRNPs, and SR proteins. Genes Dev. 29, 2168-2182. doi: $10.1101 /$ gad.270421.115

Kristensen, L. S., Andersen, M. S., Stagsted, L. V. W., Ebbesen, K. K., Hansen, T. B., and Kjems, J. (2019). The biogenesis, biology and characterization of circular RNAs. Nat. Rev. Genet. 20, 675-691.

Kristensen, L. S., Okholm, T. L. H., Venø, M. T., and Kjems, J. (2018). Circular RNAs are abundantly expressed and upregulated during human epidermal stem cell differentiation. RNA Biol. 15, 280-291. doi: 10.1080/15476286.2017. 1409931

Kumar, L., Shamsuzzama, L., Haque, R., Baghel, T., and Nazir, A. (2017). Circular RNAs: the emerging class of Non-coding RNAs and their potential role in human neurodegenerative diseases. Mol. Neurobiol. 54, 7224-7234. doi: 10. 1007/s12035-016-0213-8

Kumar, L., Shamsuzzama, O., Jadiya, P., Haque, R., Shukla, S., and Nazir, A. (2018). Functional characterization of novel circular RNA molecule, circzip-2 and its synthesizing gene zip-2 in C. elegans model of Parkinson's disease. Mol. Neurobiol. 55, 6914-6926. doi: 10.1007/s12035-018-0903-5

Kun-Peng, Z., Chun-Lin, Z., Jian-Ping, H., and Lei, Z. (2018a). A novel circulating hsa_circ_0081001 act as a potential biomarker for diagnosis and prognosis of osteosarcoma. Int. J. Biol. Sci. 14, 1513-1520. doi: 10.7150/ijbs.27523

Kun-Peng, Z., Xiao-Long, M., and Chun-Lin, Z. (2018b). Overexpressed circPVT1, a potential new circular RNA biomarker, contributes to doxorubicin and 
cisplatin resistance of osteosarcoma cells by regulating ABCB1. Int. J. Biol. Sci. 14, 321-330. doi: 10.7150/ijbs. 24360

Langmead, B., and Salzberg, S. L. (2012). Fast gapped-read alignment with Bowtie 2. Nat. Methods 9, 357-359. doi: 10.1038/nmeth.1923

Lasda, E., and Parker, R. (2016). Circular RNAs Co-precipitate with extracellular vesicles: a possible mechanism for circRNA clearance. PLoS One 11:e0148407. doi: 10.1371/journal.pone.0148407

Legnini, I., Di Timoteo, G., Rossi, F., Morlando, M., Briganti, F., Sthandier, O., et al. (2017). Circ-ZNF609 is a circular RNA that can be translated and functions in myogenesis. Mol. Cell 66, 22-37.e29.

Li, G., Yang, H., Han, K., Zhu, D., Lun, P., and Zhao, Y. (2018a). A novel circular RNA, hsa_circ_0046701, promotes carcinogenesis by increasing the expression of miR-142-3p target ITGB8 in glioma. Biochem. Biophys. Res. Commun. 498, 254-261. doi: 10.1016/j.bbrc.2018.01.076

Li, J., Li, Z., Jiang, P., Peng, M., Zhang, X., Chen, K., et al. (2018b). Circular RNA IARS (circ-IARS) secreted by pancreatic cancer cells and located within exosomes regulates endothelial monolayer permeability to promote tumor metastasis. J. Exp. Clin. Cancer Res. 37:177.

Li, J., Lin, H., Sun, Z., Kong, G., Yan, X., Wang, Y., et al. (2018c). High-throughput data of circular RNA profiles in human temporal cortex tissue reveals novel insights into temporal lobe epilepsy. Cell Physiol. Biochem. 45, 677-691. doi: $10.1159 / 000487161$

Li, J., Wang, J., Chen, Z., Chen, Y., and Jin, M. (2018d). Hsa_circ_0079530 promotes cell proliferation and invasion in non-small cell lung cancer. Gene $665,1-5$.

Li, S., Gu, H., Huang, Y., Peng, Q., Zhou, R., Yi, P., et al. (2018e). Circular RNA 101368/miR-200a axis modulates the migration of hepatocellular carcinoma through HMGB1/RAGE signaling. Cell Cycle 17, 2349-2359. doi: 10.1080/ 15384101.2018.1526599

Li, S., Li, Y., Chen, B., Zhao, J., Yu, S., Tang, Y., et al. (2018f). exoRBase: a database of circRNA, lncRNA and mRNA in human blood exosomes. Nucleic Acids Res. 46, D106-D112.

Li, X., Chu, C., Pei, J., Mãndoiu, I., and Wu, Y. (2018g). CircMarker: a fast and accurate algorithm for circular RNA detection. BMC Genomics 19:572. doi: 10.1186/s12864-018-4926-0

Li, X., Wang, J., Zhang, C., Lin, C., and Zhang, J. (2018h). Circular RNA circITGA7 inhibits colorectal cancer growth and metastasis by modulating the Ras pathway and upregulating transcription of its host gene ITGA7. J. Pathol. 246, 166-179. doi: 10.1002/path. 5125

Li, Z., Yanfang, W., Li, J., Jiang, P., Peng, T., Chen, K., et al. (2018i). Tumorreleased exosomal circular RNA PDE8A promotes invasive growth via the miR338/MACC1/MET pathway in pancreatic cancer. Cancer Lett. 432, 237-250. doi: 10.1016/j.canlet.2018.04.035

Li, L., Bu, D., and Zhao, Y. (2019). CircRNAwrap - a flexible pipeline for circRNA identification, transcript prediction, and abundance estimation. FEBS Lett. 593, 1179-1189.

Li, X., Yang, B., Ren, H., Xiao, T., Zhang, L., Li, L., et al. (2019). Hsa_circ_0002483 inhibited the progression and enhanced the Taxol sensitivity of non-small cell lung cancer by targeting miR-182-5p. Cell Death Dis. 10:953.

Li, X. N., Wang, Z. J., Ye, C. X., Zhao, B. C., Huang, X. X., and Yang, L. (2019). Circular RNA circVAPA is up-regulated and exerts oncogenic properties by sponging miR-101 in colorectal cancer. Biomed. Pharmacother. 112:108611. doi: 10.1016/j.biopha.2019.108611

Li, Y., Wan, B., Liu, L., Zhou, L., and Zeng, Q. (2019). Circular RNA circMTO1 suppresses bladder cancer metastasis by sponging miR-221 and inhibiting epithelial-to-mesenchymal transition. Biochem. Biophys. Res. Commun. 508, 991-996. doi: 10.1016/j.bbrc.2018.12.046

Li, Z., Hu, Y., Zeng, Q., Wang, H., Yan, J., Li, H., et al. (2019a). Circular RNA MYLK promotes hepatocellular carcinoma progression by increasing Rab23 expression by sponging miR-362-3p. Cancer Cell Int. 19:211.

Li, Z., Zhou, Y., Yang, G., He, S., Qiu, X., Zhang, L., et al. (2019b). Using circular RNA SMARCA5 as a potential novel biomarker for hepatocellular carcinoma. Clin. Chim. Acta 492, 37-44. doi: 10.1016/j.cca.2019.02.001

Li, X., Liu, C. X., Xue, W., Zhang, Y., Jiang, S., Yin, Q. F., et al. (2017). Coordinated circRNA Biogenesis and Function with NF90/NF110 in Viral Infection. Mol. Cell 67, 214-227.e217.
Li, Y., and Huang, S. (2017). Response to circular RNA profile identifies circPVT1 as a proliferative factor and prognostic marker in gastric cancer. Cancer Lett. 388, 208-219.

Liang, D., Tatomer, D. C., Luo, Z., Wu, H., Yang, L., Chen, L. L., et al. (2017). The output of protein-coding genes shifts to circular RNAs when the Pre-mRNA processing machinery is limiting. Mol. Cell 68, 940-954.e943.

Liang, H. F., Zhang, X. Z., Liu, B. G., Jia, G. T., and Li, W. L. (2017). Circular RNA circ-ABCB10 promotes breast cancer proliferation and progression through sponging miR-1271. Am. J. Cancer Res. 7, 1566-1576.

Liang, W. C., Wong, C. W., Liang, P. P., Shi, M., Cao, Y., Rao, S. T., et al. (2019). Translation of the circular RNA circ $\beta$-catenin promotes liver cancer cell growth through activation of the Wnt pathway. Genome Biol. 20:84.

Lidonnici, M. R., Aprile, A., Frittoli, M. C., Mandelli, G., Paleari, Y., Spinelli, A., et al. (2017). Plerixafor and G-CSF combination mobilizes hematopoietic stem and progenitors cells with a distinct transcriptional profile and a reduced in vivo homing capacity compared to plerixafor alone. Haematologica 102, e120-e124.

Lin, J., Cai, D., Li, W., Yu, T., Mao, H., Jiang, S., et al. (2019). Plasma circular RNA panel acts as a novel diagnostic biomarker for colorectal cancer. Clin. Biochem. 74, 60-68. doi: 10.1016/j.clinbiochem.2019.10.012

Lin, Z., Konno, M., Abad-Zapatero, C., Wierenga, R., Murthy, M. R., Ray, W. J., et al. (1986). The structure of rabbit muscle phosphoglucomutase at intermediate resolution. J. Biol. Chem. 261, 264-274. doi: 10.1016/s00219258(17)42464-1

Liu, C. X., Li, X., Nan, F., Jiang, S., Gao, X., Guo, S. K., et al. (2019). Structure and degradation of circular RNAs Regulate PKR activation in innate immunity. Cell $177,865-880 . e 821$.

Liu, Y. Y., Zhang, L. Y., and Du, W. Z. (2019). Circular RNA circ-PVT1 contributes to paclitaxel resistance of gastric cancer cells through the regulation of ZEB1 expression by sponging miR-124-3p. Biosci. Rep. 39:BSR20193045.

Liu, J., Kong, F., Lou, S., Yang, D., and Gu, L. (2018). Global identification of circular RNAs in chronic myeloid leukemia reveals hsa_circ_0080145 regulates cell proliferation by sponging miR-29b. Biochem. Biophys. Res. Commun. 504, 660-665. doi: 10.1016/j.bbrc.2018.08.154

Liu, J., Zhang, X., Yan, M., and Li, H. (2020). Emerging role of circular RNAs in cancer. Front. Oncol. 10:663. doi: 10.3389/fonc.2020.00663

Liu, Q., Zhang, X., Hu, X., Dai, L., Fu, X., Zhang, J., et al. (2016). Circular RNA related to the chondrocyte ECM regulates MMP13 expression by functioning as a MiR-136 'Sponge' in human cartilage degradation. Sci. Rep. 6:22572.

Lu, D., Ho, E. S., Mai, H., Zang, J., Liu, Y., Li, Y., et al. (2020). Identification of blood circular rnas as potential biomarkers for acute ischemic stroke. Front. Neurosci. 14:81. doi: $10.3389 /$ fnins. 2020.0008

Ma, J., Fang, L., Yang, Q., Hibberd, S., Du, W. W., Wu, N., et al. (2019). Posttranscriptional regulation of AKT by circular RNA angiomotin- like 1 mediates chemoresistance against paclitaxel in breast cancer cells. Aging 11, 11369-11381. doi: 10.18632/aging.102535

Ma, L., Wang, Z., Xie, M., Quan, Y., Zhu, W., Yang, F., et al. (2020). Silencing of circRACGAP1 sensitizes gastric cancer cells to apatinib via modulating autophagy by targeting miR-3657 and ATG7. Cell Death Dis. 11:169.

Maass, P. G., Glažar, P., Memczak, S., Dittmar, G., Hollfinger, I., Schreyer, L., et al. (2017). A map of human circular RNAs in clinically relevant tissues. J. Mol. Med. 95, 1179-1189. doi: 10.1007/s00109-017-1582-9

Mao, M., Hu, Y., Yang, Y., Qian, Y., Wei, H., Fan, W., et al. (2018). Modeling and predicting the activities of trans-acting splicing factors with machine learning. Cell Syst. 7, 510-520.e514.

Memczak, S., Jens, M., Elefsinioti, A., Torti, F., Krueger, J., Rybak, A., et al. (2013). Circular RNAs are a large class of animal RNAs with regulatory potency. Nature 495, 333-338. doi: 10.1038/nature 11928

Meng, J., Chen, S., Han, J. X., Qian, B., Wang, X. R., Zhong, W. L., et al. (2018). Twist1 regulates vimentin through Cul2 circular RNA to promote EMT in hepatocellular carcinoma. Cancer Res. 78, 4150-4162. doi: 10.1158/0008-5472. can-17-3009

Meng, X., Chen, Q., Zhang, P., and Chen, M. (2017). CircPro: an integrated tool for the identification of circRNAs with protein-coding potential. Bioinformatics 33, 3314-3316. doi: 10.1093/bioinformatics/btx446

Mitra, A., Pfeifer, K., and Park, K. S. (2018). Circular RNAs and competing endogenous RNA (ceRNA) networks. Transl. Cancer Res. 7, S624-S628. 
Okholm, T. L. H., Nielsen, M. M., Hamilton, M. P., Christensen, L. L., Vang, S., Hedegaard, J., et al. (2017). Circular RNA expression is abundant and correlated to aggressiveness in early-stage bladder cancer. NPJ Genom. Med. 2:36.

Pamudurti, N. R., Bartok, O., Jens, M., Ashwal-Fluss, R., Stottmeister, C., Ruhe, L., et al. (2017). Translation of CircRNAs. Mol. Cell 66, 9-21.e27.

Pan, B., Qin, J., Liu, X., He, B., Wang, X., Pan, Y., et al. (2019). Identification of serum exosomal hsa-circ-0004771 as a novel diagnostic biomarker of colorectal cancer. Front. Genet. 10:1096. doi: 10.3389/fgene.2019.01096

Pan, H., Li, T., Jiang, Y., Pan, C., Ding, Y., Huang, Z., et al. (2018). Overexpression of circular RNA ciRS-7 abrogates the tumor suppressive effect of miR-7 on gastric cancer via PTEN/PI3K/AKT signaling pathway. J. Cell Biochem. 119, 440-446. doi: 10.1002/jcb.26201

Pan, Y., Lou, J., Wang, H., An, N., Chen, H., Zhang, Q., et al. (2018). CircBA9.3 supports the survival of leukaemic cells by up-regulating c-ABL1 or BCR-ABL1 protein levels. Blood Cells Mol. Dis. 73, 38-44. doi: 10.1016/j.bcmd.2018.09.002

Panda, A. C., Grammatikakis, I., Kim, K. M., De, S., Martindale, J. L., Munk, R., et al. (2017). Identification of senescence-associated circular RNAs (SAC-RNAs) reveals senescence suppressor CircPVT1. Nucleic Acids Res. 45, 4021-4035. doi: 10.1093/nar/gkw1201

Piwecka, M., Glažar, P., Hernandez-Miranda, L. R., Memczak, S., Wolf, S. A., Rybak-Wolf, A., et al. (2017). Loss of a mammalian circular RNA locus causes miRNA deregulation and affects brain function. Science 357:eaam8526. doi: 10.1126/science.aam8526

Preußer, C., Hung, L. H., Schneider, T., Schreiner, S., Hardt, M., Moebus, A., et al. (2018). Selective release of circRNAs in platelet-derived extracellular vesicles. J. Extracell Ves. 7:1424473. doi: 10.1080/20013078.2018.1424473

Qiu, L., Huang, Y., Li, Z., Dong, X., Chen, G., Xu, H., et al. (2019). Circular RNA profiling identifies circADAMTS13 as a miR-484 sponge which suppresses cell proliferation in hepatocellular carcinoma. Mol. Oncol. 13, 441-455. doi: 10.1002/1878-0261.12424

Ren, T., Liu, C., Hou, J., and Shan, F. (2020). Hsa_circ_0043265 suppresses proliferation, metastasis, EMT and promotes apoptosis in non-small cell lung cancer through miR-25-3p/FOXP2 pathway. Oncol. Targets Ther. 13, 38673880. doi: $10.2147 /$ ott.s235231

Robic, A., Demars, J., and Kühn, C. (2020). In-Depth analysis reveals production of circular RNAs from non-coding sequences. Cells 9:1806. doi: 10.3390/ cells9081806

Rybak-Wolf, A., Stottmeister, C., Glažar, P., Jens, M., Pino, N., Giusti, S., et al. (2015). Circular RNAs in the mammalian brain are highly abundant, conserved, and dynamically expressed. Mol. Cell 58, 870-885. doi: 10.1016/j.molcel.2015. 03.027

Salzman, J., Chen, R. E., Olsen, M. N., Wang, P. L., and Brown, P. O. (2013). Cell-type specific features of circular RNA expression. PLoS Genet. 9:e1003777. doi: 10.1371/journal.pone.1003777

Salzman, J., Gawad, C., Wang, P. L., Lacayo, N., and Brown, P. O. (2012). Circular RNAs are the predominant transcript isoform from hundreds of human genes in diverse cell types. PLoS One 7:e30733. doi: 10.1371/journal.pone.030733

Shang, J., Chen, W. M., Wang, Z. H., Wei, T. N., Chen, Z. Z., and Wu, W. B. (2019). CircPAN3 mediates drug resistance in acute myeloid leukemia through the miR-153-5p/miR-183-5p-XIAP axis. Exp. Hematol. 70, 42-54.e43.

Shao, Y., Li, J., Lu, R., Li, T., Yang, Y., Xiao, B., et al. (2017). Global circular RNA expression profile of human gastric cancer and its clinical significance. Cancer Med. 6, 1173-1180. doi: 10.1002/cam4.1055

Shi, Y., Fang, N., Li, Y., Guo, Z., Jiang, W., He, Y., et al. (2020). Circular RNA LPAR3 sponges microRNA-198 to facilitate esophageal cancer migration, invasion, and metastasis. Cancer Sci. 111, 2824-2836. doi: 10.1111/cas.14511

Shi, Z., Chen, T., Yao, Q., Zheng, L., Zhang, Z., Wang, J., et al. (2017). The circular RNA ciRS-7 promotes APP and BACE1 degradation in an NF- $\kappa B-d e p e n d e n t$ manner. FEBS J. 284, 1096-1109. doi: 10.1111/febs. 14045

Shukla, S., Kavak, E., Gregory, M., Imashimizu, M., Shutinoski, B., Kashlev, M., et al. (2011). CTCF-promoted RNA polymerase II pausing links DNA methylation to splicing. Nature 479, 74-79. doi: 10.1038/nature10442

Song, X., Zhang, N., Han, P., Moon, B. S., Lai, R. K., Wang, K., et al. (2016). Circular RNA profile in gliomas revealed by identification tool UROBORUS. Nucleic Acids Res. 44:e87. doi: 10.1093/nar/gkw075

Su, H., Tao, T., Yang, Z., Kang, X., Zhang, X., Kang, D., et al. (2019). Circular RNA CTFRC acts as the sponge of MicroRNA-107 to promote bladder carcinoma progression. Mol. Cancer 18:27.
Su, M., Xiao, Y., Ma, J., Tang, Y., Tian, B., Zhang, Y., et al. (2019). Circular RNAs in Cancer: emerging functions in hallmarks, stemness, resistance and roles as potential biomarkers. Mol. Cancer 18, 90-90.

Sun, H., Tang, W., Rong, D., Jin, H., Fu, K., Zhang, W., et al. (2018). Hsa_circ_0000520, a potential new circular RNA biomarker, is involved in gastric carcinoma. Cancer Biomark. 21, 299-306. doi: 10.3233/cbm- 170379

Szabo, L., Morey, R., Palpant, N. J., Wang, P. L., Afari, N., Jiang, C., et al. (2015). Statistically based splicing detection reveals neural enrichment and tissuespecific induction of circular RNA during human fetal development. Genome Biol. 16:126.

Taïbi, F., Metzinger-Le Meuth, V., Massy, Z. A., and Metzinger, L. (2014). miR-223: an inflammatory oncomiR enters the cardiovascular field. Biochim. Biophys. Acta 1842, 1001-1009. doi: 10.1016/j.bbadis.2014.03.005

Tang, H., Huang, X., Wang, J., Yang, L., Kong, Y., Gao, G., et al. (2019). circKIF4A acts as a prognostic factor and mediator to regulate the progression of triplenegative breast cancer. Mol. Cancer 18:23.

Tang, Q., Chen, Z., Zhao, L., and Xu, H. (2019). Circular RNA hsa_circ_0000515 acts as a miR-326 sponge to promote cervical cancer progression through up-regulation of ELK1. Aging 11, 9982-9999. doi: 10.18632/aging.10 2356

Tatomer, D. C., and Wilusz, J. E. (2017). An unchartered journey for ribosomes: circumnavigating circular RNAs to produce proteins. Mol. Cell 66, 1-2. doi: 10.1016/j.molcel.2017.03.011

Verheijen, B. M., and Pasterkamp, R. J. (2017). Commentary: FUS affects circular RNA expression in murine embryonic stem cell-derived motor neurons. Front. Mol. Neurosci. 10:412. doi: 10.3389/fnmol.2017.00412

Vo, J. N., Cieslik, M., Zhang, Y., Shukla, S., Xiao, L., Zhang, Y., et al. (2019). The landscape of Circular RNA in cancer. Cell 176, 869-881.e813.

Wan, B., Hu, H., Wang, R., Liu, W., and Chen, D. (2020). Therapeutic potential of circular RNAs in osteosarcoma. Front. Oncol. 10:370. doi: 10.3389/fonc.2020. 00370

Wang, J., and Li, H. (2018). CircRNA circ_0067934 silencing inhibits the proliferation, migration and invasion of NSCLC cells and correlates with unfavorable prognosis in NSCLC. Eur. Rev. Med. Pharmacol. Sci. 22, 30533060 .

Wang, K., Gan, T. Y., Li, N., Liu, C. Y., Zhou, L. Y., Gao, J. N., et al. (2017). Circular RNA mediates cardiomyocyte death via miRNA-dependent upregulation of MTP18 expression. Cell Death Differ. 24, 1111-1120. doi: 10.1038/cdd.2017.61

Wang, M., Yu, F., Wu, W., Zhang, Y., Chang, W., Ponnusamy, M., et al. (2017). Circular RNAs: A novel type of non-coding RNA and their potential implications in antiviral immunity. Intern. J. Biol. Sci. 13, 1497-1506. doi: 10.7150/ijbs.22531

Wang, K., Singh, D., Zeng, Z., Coleman, S. J., Huang, Y., Savich, G. L., et al. (2010). MapSplice: accurate mapping of RNA-seq reads for splice junction discovery. Nucleic Acids Res. 38:e178. doi: 10.1093/nar/gkq622

Wang, L., Tong, X., Zhou, Z., Wang, S., Lei, Z., Zhang, T., et al. (2018). Circular RNA hsa_circ_0008305 (circPTK2) inhibits TGF- $\beta$-induced epithelialmesenchymal transition and metastasis by controlling TIF $1 \gamma$ in non-small cell lung cancer. Mol. Cancer 17:140.

Wang, X., Zhang, C., Wu, Z., Chen, Y., and Shi, W. (2018). CircIBTK inhibits DNA demethylation and activation of AKT signaling pathway via miR-29b in peripheral blood mononuclear cells in systemic lupus erythematosus. Arthr. Res. Ther. 20:118.

Wang, S., Zhang, Y., Cai, Q., Ma, M., Jin, L. Y., Weng, M., et al. (2019). Circular RNA FOXP1 promotes tumor progression and Warburg effect in gallbladder cancer by regulating PKLR expression. Mol. Cancer 18:145.

Wang, Y., Li, Y., He, H., and Wang, F. (2019). Circular RNA circ-PRMT5 facilitates non-small cell lung cancer proliferation through upregulating EZH2 via sponging miR-377/382/498. Gene 720:144099. doi: 10.1016/j.gene.2019. 144099

Wang, X., and Fang, L. (2018). Advances in circular RNAs and their roles in breast Cancer. J. Exp. Clin. Cancer Res. 37:206.

Wang, X., Zhang, H., Yang, H., Bai, M., Ning, T., Deng, T., et al. (2020). Exosomedelivered circRNA promotes glycolysis to induce chemoresistance through the miR-122-PKM2 axis in colorectal cancer. Mol. Oncol. 14, 539-555. doi: 10. 1002/1878-0261.12629

Wei, Y., Chen, X., Liang, C., Ling, Y., Yang, X., Ye, X., et al. (2020). A noncoding regulatory RNAs network driven by circ-CDYL acts specifically in the early 
stages hepatocellular carcinoma. Hepatology 71, 130-147. doi: 10.1002/hep. 30795

Weng, W., Wei, Q., Toden, S., Yoshida, K., Nagasaka, T., Fujiwara, T., et al. (2017). Circular RNA ciRS-7-A promising prognostic biomarker and a potential therapeutic target in colorectal cancer. Clin. Cancer Res. 23, 3918-3928. doi: 10.1158/1078-0432.ccr-16-2541

Wesselhoeft, R. A., Kowalski, P. S., and Anderson, D. G. (2018). Engineering circular RNA for potent and stable translation in eukaryotic cells. Nat. Commun. 9:2629.

Westholm, J. O., Miura, P., Olson, S., Shenker, S., Joseph, B., Sanfilippo, P., et al. (2014). Genome-wide analysis of drosophila circular RNAs reveals their structural and sequence properties and age-dependent neural accumulation. Cell Rep. 9, 1966-1980. doi: 10.1016/j.celrep.2014.10.062

Wu, D. M., Wen, X., Han, X. R., Wang, S., Wang, Y. J., Shen, M., et al. (2018). Role of Circular RNA DLEU2 in human acute myeloid leukemia. Mol. Cell Biol. 38:e00259-18.

Wu, Q., Li, P., Wu, M., and Liu, Q. (2019). Deregulation of circular RNAs in cancer from the perspectives of aberrant biogenesis, transport and removal. Front. Genet. 10:16. doi: 10.3389/fgene.2019.00016

Wu, Z., Sun, H., Li, J., and Jin, H. (2019). Circular RNAs in leukemia. Aging 11, 4757-4771. doi: 10.18632/aging.102091

Wu, Q., Wang, H., Liu, L., Zhu, K., Yu, W., and Guo, J. (2020). Hsa_circ_0001546 acts as a miRNA-421 sponge to inhibit the chemoresistance of gastric cancer cells via ATM/Chk2/p53-dependent pathway. Biochem. Biophys. Res. Commun. 521, 303-309. doi: 10.1016/j.bbrc.2019.10.117

Xia, P., Wang, S., Ye, B., Du, Y., Li, C., Xiong, Z., et al. (2018). A circular RNA protects dormant hematopoietic stem cells from DNA sensor cGAS-mediated exhaustion. Immunity 48, 688-701.e687.

Xia, S., Feng, J., Chen, K., Ma, Y., Gong, J., Cai, F., et al. (2018). CSCD: a database for cancer-specific circular RNAs. Nucleic Acids Res. 46, D925-D929.

Xia, S., Feng, J., Lei, L., Hu, J., Xia, L., Wang, J., et al. (2017). Comprehensive characterization of tissue-specific circular RNAs in the human and mouse genomes. Brief Bioinform. 18, 984-992.

Xia, W., Qiu, M., Chen, R., Wang, S., Leng, X., Wang, J., et al. (2016). Circular RNA has_circ_0067934 is upregulated in esophageal squamous cell carcinoma and promoted proliferation. Sci. Rep. 6:35576.

Xia, X., Li, X., Li, F., Wu, X., Zhang, M., Zhou, H., et al. (2019). Correction to: a novel tumor suppressor protein encoded by circular AKT3 RNA inhibits glioblastoma tumorigenicity by competing with active phosphoinositidedependent Kinase-1. Mol. Cancer 18:149.

Xiong, W., Ai, Y. Q., Li, Y. F., Ye, Q., Chen, Z. T., Qin, J. Y., et al. (2017). Microarray analysis of circular RNA expression profile associated with 5-fluorouracilbased chemoradiation resistance in colorectal cancer cells. Biomed. Res. Int. 2017:8421614.

Xu, G., Chen, Y., Fu, M., Zang, X., Cang, M., Niu, Y., et al. (2020). Circular RNA CCDC66 promotes gastric cancer progression by regulating c-Myc and TGF- $\beta$ signaling pathways. J. Cancer 11, 2759-2768. doi: 10.7150/jca.37718

Xu, Y., Yu, J., Huang, Z., Fu, B., Tao, Y., Qi, X., et al. (2020). Circular RNA hsa_circ_0000326 acts as a miR-338-3p sponge to facilitate lung adenocarcinoma progression. J. Exp. Clin. Cancer Res. 39:57.

Xu, H., Zhang, Y., Qi, L., Ding, L., Jiang, H., and Yu, H. (2018). NFIX circular RNA promotes glioma progression by regulating miR-34a-5p via notch signaling pathway. Front. Mol. Neurosci. 11:225. doi: 10.3389/fnmol.2018.00225

Xu, Y., Yao, Y., Leng, K., Ji, D., Qu, L., Liu, Y., et al. (2018). Increased expression of circular RNA circ_0005230 indicates dismal prognosis in breast cancer and regulates cell proliferation and invasion via miR-618/ CBX8 signal pathway. Cell Physiol. Biochem. 51, 1710-1722. doi: 10.1159/000495675

Yan, B., Zhang, W., Mao, X. W., and Jiang, L. Y. (2018). Circular RNA ciRS-7 correlates with advance disease and poor prognosis, and its down-regulation inhibits cells proliferation while induces cells apoptosis in non-small cell lung cancer. Eur. Rev. Med. Pharmacol. Sci. 22, 8712-8721.

Yang, F., Liu, D. Y., Guo, J. T., Ge, N., Zhu, P., Liu, X., et al. (2017). Circular RNA circ-LDLRAD3 as a biomarker in diagnosis of pancreatic cancer. World J. Gastroenterol. 23, 8345-8354. doi: 10.3748/wjg.v23.i47.8345

Yang, Y., Fan, X., Mao, M., Song, X., Wu, P., Zhang, Y., et al. (2017). Extensive translation of circular RNAs driven by N(6)-methyladenosine. Cell Res. 27, 626-641. doi: $10.1038 / \mathrm{cr} .2017 .31$
Yang, Z., Xie, L., Han, L., Qu, X., Yang, Y., Zhang, Y., et al. (2017). Circular RNAs: regulators of cancer-related signaling pathways and potential diagnostic biomarkers for human cancers. Theranostics 7, 3106-3117. doi: 10.7150/thno. 19016

Yang, H., Li, X., Meng, Q., Sun, H., Wu, S., Hu, W., et al. (2020). CircPTK2 (hsa_circ_0005273) as a novel therapeutic target for metastatic colorectal cancer. Mol. Cancer 19:13.

Yang, P., Qiu, Z., Jiang, Y., Dong, L., Yang, W., Gu, C., et al. (2016). Silencing of cZNF292 circular RNA suppresses human glioma tube formation via the Wnt/B-catenin signaling pathway. Oncotarget 7, 63449-63455. doi: 10.18632/ oncotarget.11523

Yang, Y., Gao, X., Zhang, M., Yan, S., Sun, C., Xiao, F., et al. (2018). Novel role of FBXW7 circular RNA in repressing glioma tumorigenesis. J. Natl. Cancer Inst. 110, 304-315. doi: 10.1093/jnci/djx166

Yang, Y., and Wang, Z. (2019). IRES-mediated cap-independent translation, a path leading to hidden proteome. J. Mol. Cell Biol. 11, 911-919. doi: 10.1093/jmcb/ mjz091

Yao, D., Zhang, L., Zheng, M., Sun, X., Lu, Y., and Liu, P. (2018). Circ2Disease: a manually curated database of experimentally validated circRNAs in human disease. Sci. Rep. 8:11018.

Ye, C. Y., Zhang, X., Chu, Q., Liu, C., Yu, Y., Jiang, W., et al. (2017). Full-length sequence assembly reveals circular RNAs with diverse non-GT/AG splicing signals in rice. RNA Biol. 14, 1055-1063. doi: 10.1080/15476286.2016.1245268

Yi, Y. Y., Yi, J., Zhu, X., Zhang, J., Zhou, J., Tang, X., et al. (2019). Circular RNA of vimentin expression as a valuable predictor for acute myeloid leukemia development and prognosis. J. Cell Physiol. 234, 3711-3719. doi: 10.1002/jcp. 27145

You, X., Vlatkovic, I., Babic, A., Will, T., Epstein, I., Tushev, G., et al. (2015). Neural circular RNAs are derived from synaptic genes and regulated by development and plasticity. Nat. Neurosci. 18, 603-610. doi: 10.1038/nn.3975

Yu, J., Ding, W. B., Wang, M. C., Guo, X. G., Xu, J., Xu, Q. G., et al. (2020). Plasma circular RNA panel to diagnose hepatitis B virus-related hepatocellular carcinoma: a large-scale, multicenter study. Int. J. Cancer 146, 1754-1763. doi: $10.1002 /$ ijc. 32647

Yuan, W., Zhou, R., Wang, J., Han, J., Yang, X., Yu, H., et al. (2019). Circular RNA Cdr1as sensitizes bladder cancer to cisplatin by upregulating APAF1 expression through miR-1270 inhibition. Mol. Oncol. 13, 1559-1576. doi: 10.1002/18780261.12523

Zeng, K., Chen, X., Xu, M., Liu, X., Hu, X., Xu, T., et al. (2018). CircHIPK3 promotes colorectal cancer growth and metastasis by sponging miR-7. Cell Death Dis. 9:417.

Zhang, H., Wang, G., Ding, C., Liu, P., Wang, R., Ding, W., et al. (2017). Increased circular RNA UBAP2 acts as a sponge of miR-143 to promote osteosarcoma progression. Oncotarget 8, 61687-61697. doi: 10.18632/oncotarget.18671

Zhang, X. L., Xu, L. L., and Wang, F. (2017). Hsa_circ_0020397 regulates colorectal cancer cell viability, apoptosis and invasion by promoting the expression of the miR-138 targets TERT and PD-L1. Cell Biol. Int. 41, 1056-1064. doi: 10.1002/cbin. 10826

Zhang, L., Zhou, Q., Qiu, Q., Hou, L., Wu, M., Li, J., et al. (2019). CircPLEKHM3 acts as a tumor suppressor through regulation of the miR9/BRCA1/DNAJB6/KLF4/AKT1 axis in ovarian cancer. Mol. Cancer 18:144.

Zhang, X., Wang, S., Wang, H., Cao, J., Huang, X., Chen, Z., et al. (2019). Circular RNA circNRIP1 acts as a microRNA-149-5p sponge to promote gastric cancer progression via the AKT1/mTOR pathway. Mol. Cancer 18:20.

Zhang, M., Huang, N., Yang, X., Luo, J., Yan, S., Xiao, F., et al. (2018a). A novel protein encoded by the circular form of the SHPRH gene suppresses glioma tumorigenesis. Oncogene 37, 1805-1814. doi: 10.1038/s41388-017-0019-9

Zhang, M., Zhao, K., Xu, X., Yang, Y., Yan, S., Wei, P., et al. (2018b). A peptide encoded by circular form of LINC-PINT suppresses oncogenic transcriptional elongation in glioblastoma. Nat. Commun. 9:4475.

Zhang, R., Xu, J., Zhao, J., and Wang, X. (2018c). Silencing of hsa_circ_0007534 suppresses proliferation and induces apoptosis in colorectal cancer cells. Eur. Rev. Med. Pharmacol. Sci. 22, 118-126.

Zhang, X., Xu, Y., Qian, Z., Zheng, W., Wu, Q., Chen, Y., et al. (2018d). circRNA_104075 stimulates YAP-dependent tumorigenesis through the regulation of HNF4a and may serve as a diagnostic marker in hepatocellular carcinoma. Cell Death Dis. 9:1091. 
Zhang, X., Yang, D., and Wei, Y. (2018e). Overexpressed CDR1as functions as an oncogene to promote the tumor progression via miR-7 in non-small-cell lung cancer. Oncol. Targets Ther. 11, 3979-3987. doi: 10.2147/ott.s158316

Zhang, S., Cheng, J., Quan, C., Wen, H., Feng, Z., Hu, Q., et al. (2020). circCELSR1 (hsa_circ_0063809) contributes to paclitaxel resistance of ovarian cancer cells by regulating FOXR2 expression via miR-1252. Mol. Ther. Nucleic Acids 19, 718-730. doi: 10.1016/j.omtn.2019.12.005

Zhang, X. O., Wang, H. B., Zhang, Y., Lu, X., Chen, L. L., and Yang, L. (2014). Complementary sequence-mediated exon circularization. Cell 159, 134-147. doi: 10.1016/j.cell.2014.09.001

Zhang, X. Q., and Yang, J. H. (2018). Discovering circRNA-microRNA Interactions from CLIP-Seq Data. Methods Mol. Biol. 1724, 193-207. doi: 10.1007/978-14939-7562-4_16

Zhao, X., Cai, Y., and Xu, J. (2019). Circular RNAs: biogenesis, mechanism, and function in human cancers. Int. J. Mol. Sci. 20:3926. doi: 10.3390/ijms20163926

Zhao, Z., Ji, M., Wang, Q., He, N., and Li, Y. (2019). Circular RNA Cdr1as upregulates SCAI to suppress cisplatin resistance in ovarian cancer via miR1270 suppression. Mol. Ther. Nucleic Acids 18, 24-33. doi: 10.1016/j.omtn.2019. 07.012

Zheng, F., and Xu, R. (2020). CircPVT1 contributes to chemotherapy resistance of lung adenocarcinoma through miR-145-5p/ABCC1 axis. Biomed. Pharmacother. 124:109828. doi: 10.1016/j.biopha.2020.109828

Zheng, Q., Bao, C., Guo, W., Li, S., Chen, J., Chen, B., et al. (2016). Circular RNA profiling reveals an abundant circHIPK 3 that regulates cell growth by sponging multiple miRNAs. Nat. Commun. 7:11215.

Zheng, X., Chen, L., Zhou, Y., Wang, Q., Zheng, Z., Xu, B., et al. (2019). A novel protein encoded by a circular RNA circPPP1R12A promotes tumor pathogenesis and metastasis of colon cancer via Hippo-YAP signaling. Mol. Cancer 18:47.

Zheng, Y., Ji, P., Chen, S., Hou, L., and Zhao, F. (2019). Reconstruction of full-length circular RNAs enables isoform-level quantification. Genome Med. $11: 2$.

Zhong, Z., Huang, M., Lv, M., He, Y., Duan, C., Zhang, L., et al. (2017). Circular RNA MYLK as a competing endogenous RNA promotes bladder cancer progression through modulating VEGFA/VEGFR2 signaling pathway. Cancer Lett. 403, 305-317. doi: 10.1016/j.canlet.2017.06.027
Zhong, Z., Lv, M., and Chen, J. (2016). Screening differential circular RNA expression profiles reveals the regulatory role of circTCF $25-\mathrm{miR}-103 \mathrm{a}-3 \mathrm{p} / \mathrm{miR}$ 107-CDK6 pathway in bladder carcinoma. Sci. Rep. 6:30919.

Zhou, C., Molinie, B., Daneshvar, K., Pondick, J. V., Wang, J., Van Wittenberghe, N., et al. (2017). Genome-wide maps of m6A circRNAs identify widespread and cell-type-specific methylation patterns that are distinct from mRNAs. Cell Rep. 20, 2262-2276. doi: 10.1016/j.celrep.2017.08.027

Zhou, J., Zhang, S., Chen, Z., He, Z., Xu, Y., and Li, Z. (2019). CircRNA-ENO1 promoted glycolysis and tumor progression in lung adenocarcinoma through upregulating its host gene ENO1. Cell Death Dis. 10:885.

Zhou, L. H., Yang, Y. C., Zhang, R. Y., Wang, P., Pang, M. H., and Liang, L. Q. (2018). CircRNA_0023642 promotes migration and invasion of gastric cancer cells by regulating EMT. Eur. Rev. Med. Pharmacol. Sci. 22, 22972303.

Zhu, X., Wang, X., Wei, S., Chen, Y., Chen, Y., Fan, X., et al. (2017). hsa_circ_0013958: a circular RNA and potential novel biomarker for lung adenocarcinoma. FEBS J. 284, 2170-2182. doi: 10.1111/febs.14132

Zong, Z. H., Du, Y. P., Guan, X., Chen, S., and Zhao, Y. (2019). CircWHSC1 promotes ovarian cancer progression by regulating MUC1 and hTERT through sponging miR-145 and miR-1182. J. Exp. Clin. Cancer Res. 38:437.

Zuo, L., Zhang, L., Zu, J., Wang, Z., Han, B., Chen, B., et al. (2020). Circulating circular RNAs as biomarkers for the diagnosis and prediction of outcomes in acute ischemic stroke. Stroke 51, 319-323. doi: 10.1161/strokeaha.119.027348

Conflict of Interest: The authors declare that the research was conducted in the absence of any commercial or financial relationships that could be construed as a potential conflict of interest.

Copyright (C) 2021 Nisar, Bhat, Singh, Karedath, Rizwan, Hashem, Bagga, Reddy, Jamal, Uddin, Chand, Bedognetti, El-Rifai, Frenneaux, Macha, Ahmed and Haris. This is an open-access article distributed under the terms of the Creative Commons Attribution License (CC BY). The use, distribution or reproduction in other forums is permitted, provided the original author(s) and the copyright owner(s) are credited and that the original publication in this journal is cited, in accordance with accepted academic practice. No use, distribution or reproduction is permitted which does not comply with these terms. 